\title{
A validation of Emotiv EPOC Flex saline for EEG and ERP research
}

\author{
Nikolas S Williams ${ }^{\text {Corresp., }}{ }^{1}$, Genevieve M McArthur ${ }^{1}$, Bianca de Wit $^{1}{ }^{1}$, George Ibrahim ${ }^{1}$, Nicholas A Badcock ${ }^{1,2}$ \\ 1 Department of Cognitive Science, Macquarie University, Sydney, New South Wales, Australia \\ 2 School of Psychological Science, University of Western Australia, Perth, Western Australia, Australia \\ Corresponding Author: Nikolas S Williams \\ Email address: nikolas.williams@mq.edu.au
}

Background. Previous work has validated consumer-grade electroencephalography (EEG) systems for use in research. Systems in this class are cost-effective and easy to set-up and can facilitate neuroscience outside of the laboratory. The aim of the current study was to determine if a new consumer-grade system, the Emotiv EPOC Saline Flex, was capable of capturing research-quality data.

Method. The Emotiv system was used simultaneously with a research-grade EEG system, Neuroscan Synamps2, to collect EEG data across 16 channels during five well-established paradigms: 1) a mismatch negativity (MMN) paradigm that involved a passive listening task in which rare deviant $(1500 \mathrm{~Hz})$ tones were interspersed amongst frequent standard tones $(1000 \mathrm{~Hz})$, with instructions to ignore the tones while watching a silent movie; (2) a P300 paradigm that involved an active listening task in which participants were asked to count rare deviant tones presented amongst frequent standard tones; (3) an N170 paradigm in which participants were shown images of faces and watches and asked to indicate whether the images were upright or inverted; (4) a steady-state visual evoked potential (SSVEP) paradigm in which participants passively viewed a flickering screen (15 $\mathrm{Hz}$ ) for two minutes; and (5) a resting state paradigm in which participants sat quietly with their eyes open and then closed for three minutes each.

Results. The MMN components and P300 peaks were equivalent between the two systems (BF10 $=0.25$ and BF10 $=0.26$, respectively), with high intraclass correlations (ICCs) between the ERP waveforms (>0.81). Although the N170 peak values recorded by the two systems were different (BF10 $=35.88)$, ICCs demonstrated that the N170 ERP waveforms were strongly correlated over the right hemisphere ( $P 8 ; r=0.87$ to 0.97 ), and moderatelyto-strongly correlated over the left hemisphere (P7; 0.52 to 0.84 ). For the SSVEP, the signal-to-noise ratio (SNR) was larger for Neuroscan than Emotiv EPOC Flex (19.94 vs. 8.98$, BF10 $=51764)$, but SNR z-scores indicated a significant brain response at the stimulus frequency for both Neuroscan $(z=12.47)$ and Flex $(z=11.22)$. In the resting 
state task, both systems measured similar alpha power $(\mathrm{BF} 10=0.28)$ and higher alpha power when the eyes were closed than open (BF10 $=32.27)$.

Conclusions. The saline version of the Emotiv EPOC Flex captures data similar to that of a research-grade EEG system. It can be used to measure reliable auditory and visual research-quality ERPs. In addition, it can index SSVEP signatures and is sensitive to changes in alpha oscillations. 


\section{A validation of Emotiv EPOC Flex saline for EEG and ERP research}

Nikolas S. Williams ${ }^{1}$, Genevieve McArthur ${ }^{1}$, Bianca de Wit ${ }^{1}$, George lbrahim ${ }^{1}$, Nicholas

A. Badcock ${ }^{1-2}$

${ }^{1}$ Department of Cognitive Science, Macquarie University, Sydney, Australia

${ }^{2}$ School of Psychological Science, University of Western Australia, Perth, Australia

8

9

10

11 Corresponding Author:

12 Nikolas Williams ${ }^{1}$

1316 University Ave

14 Australian Hearing Hub Level 3

15 Macquarie University, NSW 2109 Australia

16 Email address: nikolas.williams@mq.edu.au 
Abstract

19 Background. Previous work has validated consumer-grade electroencephalography

20 (EEG) systems for use in event-related potential (ERP) research. Systems in this class

21 are cost-effective and easy to set-up and can facilitate neuroscience outside of the

22 laboratory. The aim of the current study was to determine if a new consumer-grade

23 system, the Emotiv EPOC Saline Flex, was capable of capturing research-quality ERP

24 data.

25 Method. The Emotiv system was used simultaneously with a research-grade EEG

26 system, Neuroscan Synamps ${ }^{2}$, to collect EEG data across 16 channels during five well-

27 established paradigms: 1) a mismatch negativity (MMN) paradigm that involved a

28 passive listening task in which rare deviant $(1500 \mathrm{~Hz})$ tones were interspersed amongst

29 frequent standard tones $(1000 \mathrm{~Hz})$, with instructions to ignore the tones while watching

30 a silent movie; (2) a P300 paradigm that involved an active listening task in which

31 participants were asked to count rare deviant tones presented amongst frequent

32 standard tones; (3) an N170 paradigm in which participants were shown images of

33 faces and watches and asked to indicate whether the images were upright or inverted;

34 (4) a steady-state visual evoked potential (SSVEP) paradigm in which participants

35 passively viewed a flickering screen $(15 \mathrm{~Hz})$ for two minutes; and (5) a resting state

36 paradigm in which participants sat quietly with their eyes open and then closed for three

37 minutes each.

38 Results. The MMN components and P300 peaks were equivalent between the two 39 systems $\left(\mathrm{BF}_{10}=0.25\right.$ and $\mathrm{BF}_{10}=0.26$, respectively $)$, with high intraclass correlations 40 (ICCs) between the ERP waveforms (> 0.81). Although the N170 peak values recorded 
41 by the two systems were different $\left(\mathrm{BF}_{10}=35.88\right)$, ICCs demonstrated that the $\mathrm{N} 170$

42 ERP waveforms were strongly correlated over the right hemisphere (P8; $r=0.87$ to

43 0.97), and moderately-to-strongly correlated over the left hemisphere (P7; 0.52 to 0.84 ).

44 For the SSVEP, the signal-to-noise ratio (SNR) was larger for Neuroscan than Emotiv

45 EPOC Flex (19.94 vs. 8.98, $\left.\mathrm{BF}_{10}=51764\right)$, but SNR z-scores indicated a significant

46 brain response at the stimulus frequency for both Neuroscan $(z=12.47)$ and Flex $(z=$

47 11.22). In the resting state task, both systems measured similar alpha power $\left(\mathrm{BF}_{10}=\right.$

$480.28)$ and higher alpha power when the eyes were closed than open $\left(B F_{10}=32.27\right)$.

49 Conclusions. The saline version of the Emotiv EPOC Flex captures data similar to that 50 of a research-grade EEG system. It can be used to measure reliable auditory and visual 51 research-quality ERPs. In addition, it can index SSVEP signatures and is sensitive to 52 changes in alpha oscillations. 
Brain activity creates changes in electrical potentials. The pattern of these changes, or oscillations, can be recorded from sensors placed on the scalp and results

57 in an electroencephalogram (EEG; Nunez \& Srinivasan, 2006). EEG is a powerful method for investigating cognitive phenomena as it can non-invasively index neural processes that are too fleeting to be measured using other brain-imaging techniques. EEG can be analysed according to how often oscillations occur or when oscillations occur. The first approach is considered spectrum analyses in which oscillations are characterised according to their frequencies, with specific frequency bands denoted by particular terms. For example, the delta band is the slowest-wave frequency (less than 4 $\mathrm{Hz}$ ) and is typically observed during deep sleep (Amzica \& Steriade, 1998), whereas the alpha band $(8-12 \mathrm{~Hz})$ has been associated with myriad processes including attention (e.g., Ray \& Cole, 1985; Sauseng et al., 2005; Uusberg, Uibo, Kreegipuu, \& Allik, 2013),

67 relaxation (e.g., Jacobs \& Friedman, 2004), and listening effort (e.g., McMahon et al., 2016). Changes in EEG frequencies can be used to investigate clinical phenomena such as depression (e.g., Debener et al., 2000) or Alzheimer's disease (see Vecchio et al., 2013, for a review), as well as cognitive processes such as memory and attention

71 (e.g., Bonnefond \& Jensen, 2012; Düzel, Penny, \& Burgess, 2010; Klimesch, 1999).

72 Even simple resting state paradigms, in which frequency bands are compared between

73 'eyes open' and 'eyes closed' conditions, can answer questions concerning psychiatric 74 disorders (see Newson \& Thiagarajan, 2019 for a review), autism (e.g., Billeci et al., 75 2013), and ageing (e.g., Barry \& De Blasio, 2017). 
In contrast to the frequency of brain waves, EEG research is often concerned

77 with the timing of the brain's response to specific stimuli. This can be represented by event-related potentials (ERPs) which are the average waveform of electrical activity

79

80

81

82

83

84

85

86

87

that occurs immediately before and after a stimulus of interest. An auditory ERP is the average response to a particular sound (e.g., a tone) that is typically characterised by a waveform with three positive peaks, P100, P200, and P300, and two negative peaks, N100 and N200 (Luck, 2014). Differences in these waveforms between conditions or populations can indicate differences in auditory processing. For example, changes in auditory stimuli, such as the onset of a high-pitched "deviant" tone after a sequence of low-pitched "standard" tones, will generally elicit a change in neural response - even if a participant is not actively attending to the tones. Subtracting the response to the deviant tone from the standard tone reveals the mismatch negativity (MMN) component, which reflects an automatic brain response to change detection in passive auditory processing (see Garrido, Kilner, Stephan, \& Friston, 2009; Näätänen, Paavilainen, Rinne, \& Alho, 2007 for reviews).

ERPs can also be used to study higher-level cognitive processing. For example, when a participant is asked to actively attend to a sequence of standard and deviant tones, the onset of the deviant tone will produce a particularly large third peak in the auditory ERP. This P300 (or P3) response is thought to be reflective of higher-level cognitive processing such as working memory (Luck, 1998; Vogel \& Luck, 2002; Vogel, Luck, \& Shapiro, 1998) and attention (see Polich, 2007; Polich \& Kok, 1995 for reviews). The robust and distinctive nature of a P300 makes it a useful signature for investigating cognitive processing in target-detection tasks. 
ERPs can also be used to investigate cognitive processing in the visual domain.

100 For example, the face-sensitive N170 occurs when a participant is visually-presented

101 with an object. Participants will typically exhibit an ERP waveform with a negative

102 deflection occurring approximately $170 \mathrm{~ms}$ after the presentation. This deflection is

103 generally larger for faces than other objects (Eimer, 2012). In addition, the N170 is also

104 sensitive to structural changes in faces, such as a delay in onset when faces are

105 inverted (Itier, Latinus, \& Taylor, 2006; Itier \& Taylor, 2004; Rossion et al., 1999) and an

106 enhanced amplitude when faces show emotion (see Hinojosa, Mercado, \& Carretié,

1072015 for a meta-analysis).

108

Related to both frequency-based EEG studies and ERPs are steady-state visual

109 evoked potentials (SSVEPs). Like ERPs, SSVEPs are produced as a result of external

110 stimuli. Unlike ERPs, they are analysed on the frequency, rather than time, domain (see

111 Norcia, Appelbaum, Ales, Cottereau, \& Rossion, 2015 for a review) and represent a

112 phasic response to periodic stimulus, such as a flickering screen. SSVEPs will generally

113 produce a large EEG signature at the same frequency as the stimulus, making them

114 useful for investigating cognitive processes such as reading (e.g., Lochy, Van Belle, \&

115 Rossion, 2015), face perception (e.g., Liu-shuang, Norcia, \& Rossion, 2014), or to

116 characterise mental state or intent in brain-computer interfaces (e.g., Alamdari et al.,

117 2016). For example, Liu-Shuang et al. (2014) presented participants with a rapid series

118 of faces in which every fifth face was different from the others. Not only did they report

119 typical SSVEPs at the stimulus frequency, but also a larger SSVEP at the odd-face

120 frequency, suggesting participants' brains could discriminate the odd face even if the

121 participants were unaware that the faces were different. 
As they covertly measure neural processes, EEG and ERP studies are

123 particularly useful in populations for whom laboratory conditions may be inappropriate or

124 untenable. For example, both auditory and visual ERPs may be used to investigate

125 cognitive processing in schizophrenia (Feuerriegel, Churches, Hofmann, \& Keage,

126 2015), dyslexia (McArthur, Atkinson, \& Ellis, 2009; Peter, McCollum, Daliri, \&

127 Panagiotides, 2019), autism (Kim, Grammer, Benrey, Morrison, \& Lord, 2018; Schwartz,

128 Shinn-Cunningham, \& Tager-Flusberg, 2018) or neurodegeneration (Morrison,

129 Rabipour, Taler, Sheppard, \& Knoefel, 2019; Swords, Nguyen, Mudar, \& Llano, 2018).

130 This highlights three drawbacks of traditional EEG systems (e.g., Neuroscan): 1) the

131 involved setup procedures, which often include hair-washing and brushing, scalp

132 abrasion, and the application of messy gels; 2) the limitation to lab-based research, as

133 many research-grade systems are composed of large headboxes and amplifiers and

134 transmit data via physical cables; and 3) cost, as research-grade systems often cost

135 tens of thousands of dollars, putting them out of reach for many under-funded

136 researchers. Consumer-grade EEG systems offer a potential solution to these issues.

137 Several companies have released relatively inexpensive EEG systems aimed at the

138 gaming and neurofeedback market that are typically portable and much simpler to setup

139 up than research-grade systems. For example, Emotiv EPOC $®$, Muse2, and NeuroSky

140 MindWave all cost less than $\$ 700$ USD, are wireless, and use either dry electrodes or

141 saline-soaked felt pads as the conduction medium. This contrasts with research-grade

142 systems that cost much more and often use metal sensors that require the application

143 of electro-conductive gel. In addition, research-grade systems often require heavy

144 amplifiers that limit their portability. 
Over the last decade, consumer EEG systems have attracted the attention of

146 researchers. For example, a recent scoping review of Emotiv EPOC applications found

147382 journal articles and conference proceedings published from $2009-2019$, including

14851 experimental research studies and 31 validation studies (Williams, McArthur, \&

149 Badcock, 2020). Debener et al. (2012) were among the first to investigate the utility of

150 EPOC when they removed the amplifier from its original case and attached it to

151 research-quality electrodes that were inserted into an EasyCap $®$ (Herrsching,

152 Germany). They then collected data from participants in both lab and outdoor (walking

153 through a university campus) settings, with results demonstrating that the modified

154 setup measured quality auditory ERPs. In 2014, some of these same researchers

155 compared their modified EPOC setup to a research-grade system and found that P300

156 signatures were similar, suggesting that EPOC could capitalise on P300s for brain-

157 computer interface applications (De Vos, Kroesen, Emkes, \& Debener, 2014).

158 Other validations of the production-version EPOC were also published, with

159 Duvinage et al. (2013) among the first to provide evidence that it could capture auditory

160 P300 ERPs. In the same year, Badcock et al. (2013) reported that EPOC could be used

161 to measure research-quality auditory ERPs in adults. Two years later, they replicated

162 this in children (Badcock et al., 2015) and also validated the use of EPOC to measure

163 the face-sensitive N170 (de Lissa, Sörensen, Badcock, Thie, \& Mcarthur, 2015). In

164 2017, Barham et al. (2017) reported that the EPOC could be used to measure auditory

165 ERPs in adults and Melnik et al. (2017) expanded that use to SSVEPs.

Other consumer-grade EEG devices have also been validated for experimental

167 research. For example, Krigolson et al. (2017) demonstrated that a Muse EEG system 
168 could be used to measure N200 and P300 ERPs in a visual oddball task and Ratti et al.

169 (2017) showed that Muse and Mindwave could collect satisfactory resting state data in a

170 clinical setting.

$171 \quad$ Although easy-to-setup consumer-grade systems can collect research-quality

172 data, one of their biggest shortcomings is limited sensor density. For example,

173 Neurosky (1 channel), Muse2 (four channels), and EPOC (14 channels) have a far

174 fewer sensors than most research-grade EEG systems (32, 64, or 128 channels).

175 Further, the sensors of EPOC+, Muse2 and Mindwave are fixed in place (though see

176 Debener et al., 2012 and De Vos et al., 2014 wherein researchers modified an EPOC to

177 be configurable). Both the limited sensor count and rigid nature of the array limits the

178 use of these devices to certain EEG or ERP paradigms. For example, auditory ERPs, 179 such as MMN and P300, are often largest over central sites (Fz and Pz; Garrido et al., 180 2009; Näätänen et al., 2007). Data collection at these sites is not possible with EPOC, 181 Neruosky, or Muse2.

182 Recently, Emotiv released a new product, the Emotiv EPOC Flex (hereafter

183 referred to as Flex) that provides greater sensor coverage (up to 32 channels)

184 compared to other Emotiv products. In addition, the channel array is user-configurable 185 according to the $10-20$ international system, allowing researchers to optimise their EEG 186 data collection for some paradigms. Another benefit of Flex over the other consumer187 grade EEG devices mentioned is 14-bit resolution which, theoretically, provides greater 188 signal-to-noise ratios compared to lower resolution devices such as Muse 2 (10-bit) and 189 Mindwave (12-bit). These benefits of Flex are achieved while still maintaining the low 190 cost and portability for which this device classes is known. The saline sensor version of 
191 Flex also eliminates the need for electroconductive gel, which may not be desirable for

192 certain populations or in settings outside of the laboratory. See Table 1 for a

193 comparison of specifications of EEG devices.

$194 \quad$ Although Flex may fill a gap between expense, convenience, configurability, and 195 sensor coverage, its capabilities as a research system are yet to be validated against a 196 research-grade system. In this study, we compared Flex to a research-grade EEG

197 system, Neuroscan SynAmps², using a simultaneous setup across five research 198 paradigms: the auditory MMN, the auditory P300, the face-sensitive N170, the SSVEP, 199 and a resting state task.

200 Methods

201 The methods used to test participants were approved by the Macquarie 202 University Human Research Ethics Committee (Ref: 5201831203493).

203 Participants

204

We used a Bayesian stopping rule to determine participant numbers. Our pre205 registered plan (https://osf.io/b764p) was to calculate Bayes factors for a critical 206 comparison for each task after we had tested 20 participants, and then for each 207 participant thereafter, stopping each task when the Bayes factor was less than 0.33 208 (substantial evidence that the systems were equivalent; Jarosz \& Wiley, 2014) or 209 exceeded 3.00 (substantial evidence that the systems were different; Jarosz \& Wiley, 210 2014).

211 For the auditory ERP tasks, we tested 17 females and 3 males from 18 to 52 212 years of age $(M=21.4 ; S D=7.7)$ using the $M M N$ paradigm, and 18 females and 2 213 males from 18 to 26 years of age $(M=19.25$; $S D=1.9)$ for the $P 300$ paradigm. For the 
$214 \mathrm{~N} 170$, we tested 17 females and 3 males from 18 to 52 years of age $(M=21.4 ; S D=$

2157.7 ), and for the SSVEP paradigm, we tested 18 females and 2 males from 18 to 52

216 years of age $(M=20.9 ; S D=7.6)$. For the resting state paradigm, we tested 18 females

217 and 2 males from 18 to 52 years of age $(M=21.3$; $S D=7.7)$. The participants

218 characteristics differed between some tasks because we excluded participants from

219 some tasks but not others. Excluded participants were replaced with new participants.

220 See Table 2 for excluded participants and reasons for exclusions. All participants gave

221 written informed consent to be involved in the research.

222 Stimuli

223 Stimuli and instructions were presented using Psychtoolbox (version 3.0.14;

224 Brainard, 1997; Kleiner et al., 2007; Pelli, 1997) with MATLAB (version R2017b).

225 MMN paradigm. The stimuli - 666 tones - were delivered using Phillips

226 SHS4700/37 ear-clip headphones. Eight-five percent (566) were standard tones (1000

$227 \mathrm{~Hz} ; 175-\mathrm{ms}$ duration; 15-ms rise and fall time) and 15\% (100) were deviant tones (1500

$228 \mathrm{~Hz}$; $175-\mathrm{ms}$ duration; $15-\mathrm{ms}$ rise and fall time). To ensure that deviant tones were

229 distributed throughout the experiment, ten blocks were created. An equal number of

230 deviant tones (i.e., 10) were assigned to each block and then tones were presented

231 pseudo-randomly such that each block began with at least 3 successive standard tones

232 and there were at least 5 (and no more than 35) standard tones between each of the

233 deviant tones. Blocks were presented consecutively with no breaks between them. The

234 stimulus-onset asynchrony (SOA) was randomly jittered between 900 and 1100 ms to

235 minimise stimulus anticipation effects. Participants were instructed to ignore the tones 
236 and watch a silent movie on an iPad. Thus, the MMN paradigm was considered a

237 passive listening task and lasted approximately 11 minutes (666 seconds).

P300 paradigm. This paradigm was the same as the MMN paradigm except that

239 participants were instructed to count and report the number higher-pitched (i.e., deviant)

240 tones at the end of the session. Thus, the P300 paradigm was considered an active

241 listening task.

N170 paradigm. Visual stimuli were viewed on an AOC 27-inch LCD monitor

243 with a $60 \mathrm{~Hz}$ refresh rate at a distance of $50 \mathrm{~cm}$ from the participant. Stimuli were the

244 same as the previous N170 validation study (de Lissa et al., 2015), which consisted of

24575 unique face images (37 female faces, 38 male faces) and 75 unique watch images.

246 Images were cropped using a standard-sized oval such that only internal face parts

247 were visible. Each image was presented in both an upright or an inverted orientation

248 condition. Thus, there were a total of 300 images presented across four conditions

249 (upright faces, inverted faces, upright watches, inverted watches).

250 Each of 300 trials began with a white fixation cross presented for 500 ms against

251 a dark background in the centre of the screen. This was followed by presentation of the

252 face or watch stimulus for $200 \mathrm{~ms}$. A blank screen was then presented until the

253 participant indicated whether the image was upright or inverted (using the computer

254 keyboard left and right arrows). After their response, a screen was presented which

255 instructed the participants that they could blink their eyes. This blink screen lasted 1500

$256 \mathrm{~ms}$ and was followed by a 500-ms blank screen before the next trial began. Participants

257 were given a self-timed break halfway through the trials. The order of stimulus 
258 presentation was randomised at the outset of each experimental session and

259 participants completed two blocks of 150 trials with a break between blocks.

SSVEP paradigm. The stimulus was a flickering screen that oscillated between

261 grey (RGB values 160, 160, 160) and black (RGB values 0, 0, 0). The flickering screen

262 was presented on the same monitor and with the same participant setup as the N170

263 paradigm. Our goal was to create a stimulus frequency near $15 \mathrm{~Hz}$. Interactions

264 between hardware and software meant that the measured frequency was $14.36 \mathrm{~Hz}$.

265 Participants watched the flickering screen for two minutes.

266 Resting state paradigm. In the eyes open condition participants were instructed

267 to sit quietly and gaze at a black fixation cross against a grey (RGB values 160,160 ,

268 160) background. In the eyes closed condition participants were instructed to sit quietly

269 with their eyes closed until they heard a tone (which indicated the end of the trial). Each

270 trial conditions lasted three minutes.

\section{Neuroscan EEG system}

272 We used $18 \mathrm{Ag}-\mathrm{AgCl}$ electrodes for the Neuroscan setup (SynAmps RT,

273 Compumedics). These matched the Flex array and were placed in custom-cut holes

274 immediately adjacent to each of the corresponding Flex electrodes along an axis toward

$275 \mathrm{Cz}$ (for photograph of setup, see supplementary materials). Thus, the electrodes were

276 located at Fp1, F3, FT7, CP3, P7, O1, O2, P8, CP4, FT8, F4, Fp2, Fz, Cz, Pz, Oz, left

277 earlobe (online reference), and right earlobe (offline reference; see Figure 1). Vertical

278 electrooculogram (VEOG) electrodes were placed above and below the left eye and

279 horizontal electrooculogram (HEOG) electrodes were placed at the outer canthus of

280 each eye and the ground electrode was position between FPz and Fz. The Neuroscan 
281 data were sampled at $1000 \mathrm{~Hz}$ and recorded to Curry software (version 7.0.10).

282 Triggers were generated by the MATLAB stimulus-presentation scripts and inserted via 283 parallel port triggers, generated using the io64 plugin (Schieber, 2018).

284 Emotiv EPOC Flex EEG system

285

The Flex EEG electrode array consists of $32 \mathrm{Ag}-\mathrm{AgCl}$ electrodes, with 16

286 electrodes terminating in a "left" wiring harness and 16 electrodes terminating in a "right" 287 wiring harness. In addition, there is a common-mode sensor (CMS; left side) and driven288 right-leg (DRL; right side) sensor. The CMS and DRL serve as the online reference in 289 "active" sensor EEG setups like Flex. All electrodes accept saline-soaked felt pads and 290 are attached to an EasyCap $®$ (Herrsching, Germany) which is configurable according to 291 the international $10-20$ system.

292 To facilitate ease of set-up and clean-up, we modified the Flex system by 293 removing eight unused electrodes from each side. The left-side sensors (and their 294 positions) that we used were: A (Fp1), B (FT7), C (P7), D (O1), E (F3), F (CP3), G (Pz), $295 \mathrm{H}(\mathrm{Oz})$, and CMS (left earlobe). The right-side sensors (and their positions) that we 296 used were: A (Fp2), B (FT8), C (P8), D (O2), E (F4), F (CP4), G (Fz), H (Cz), and DRL 297 (right earlobe). See Figure 1 for electrode locations.

298 Flex has in-built EEG data pre-processing including a high-pass filter of $0.2 \mathrm{~Hz}$ 299 and a low-pass filter of $45 \mathrm{~Hz}$, digitisation at $1024 \mathrm{~Hz}$ and filtering using a digital $5^{\text {th }}$ 300 order sinc filter, and downsampling to $128 \mathrm{~Hz}$. EEG data were then collected using 301 Emotiv Pro (version 1.8.1).

Event-marking. One limitation of Flex is that it is not capable of native hardware 303 event-marking. To time-lock stimuli to EEG data, we used another Emotiv product, 
304 Extender (emotiv.com/extender). Extender is a hardware unit which has multiple

305 functions: 1) It can be provide auxiliary power for Emotiv products; 2) It can record EEG

306 data directly to a steady-state storage card; and 3) It can receive event-marking triggers

307 via a $2.5 \mathrm{~mm}$ tip-ring sleeve audio jack. Using function 3 , we delivered triggers to

308 Extender via a BNC adaptor and coaxial cable. Triggers were then incorporated into the

309 EEG data stream (see Figure 2).

310 Procedure

311 After cleaning the relevant areas on the face and earlobes, Neuroscan HEOG,

312 VEOG, online reference (left earlobe), and offline reference (right earlobe) electrodes

313 were attached to the participants. Electro-conductive gel was then inserted into each

314 electrode. Next participants' scalp was firmly combed to reduce electrode impedances

315 (Mahajan \& McArthur, 2010). Then the EasyCap, with all Neuroscan and Flex

316 electrodes pre-fitted was placed on the participant. The experimenter then applied gel to

317 each of the Neuroscan electrodes, taking care to first gently rub the tip of the blunt

318 metal applicator syringe on the skin of the application site $3-4$ times. Once all

319 Neuroscan electrodes had been filled with gel, the experimenter applied 2-3 mL of

320 saline solution to each of the Flex electrodes. Reference electrodes were attached to

321 both the front (Flex; CMS and DRL) and back (Neuroscan; online reference and offline

322 reference) of the earlobes. When all electrodes had been treated with a conductive

323 agent, the impedances were measured. Neuroscan impedances were measured using

324 the Curry software and adjustments to the gel were made until each electrode exhibited

325 less than $5 \mathrm{k} \Omega$. Using the Emotiv Pro software visualisation, we adjusted Flex 
326 electrodes until they were "green", which indicates impedance values below $20 \mathrm{k} \Omega$ (G.

327 Mackellar, personal communication, April 2020).

Offline EEG General Processing. All EEG data were processed using MATLAB and EEGLAB version 2019.0 (Delorme \& Makeig, 2004). Spectrum analysis on SSVEP and resting state data was performed using MATLAB and Fieldtrip (version 20190819; Oostenveld, Fries, Maris, \& Schoffelen, 2011). All processing code is available at Open Science Framework (https://osf.io/zj3f5/).

All EEG data were first bandpass filtered from 0.1 to $30 \mathrm{~Hz}$. Neuroscan data were 334 then downsampled to $129 \mathrm{~Hz}$ to match Flex data (see below regarding Flex sampling rate). Data were then re-referenced to the common average before continuing with paradigm-specific processing.

Flex sampling rate. Although the advertised sampling rate of Emotiv EPOC Flex is $128 \mathrm{~Hz}$, practical use has suggested that sampling rates can vary from device to device. As variability in sampling rates can impact the ability to accurately measure EEG data, we measured the actual sampling rate of our particular Flex device. We did 341 this by recording from the device for approximately 15 minutes. Using a custom-written 342 script in MATLAB (available at Open Science Framework; https://osf.io/zj3f5), we then 343 checked for any dropped samples (there were none) and calculated sampling rate as 344 the number of samples collected divided by the total elapsed time in seconds. This 345 resulted in a sampling rate of $129.05 \mathrm{~Hz}$. We used this figure for all further calculations 346 and analyses.

$347 \quad M M N$ processing. Following filtering, downsampling, and re-referencing to the 348 common average, we removed eye-blink artefacts using independent component 
349 analysis (ICA) in EEGLAB ('eeg_runica' function). The pruned data were then epoched 350 from $-100 \mathrm{~ms}$ to $700 \mathrm{~ms}$ relative to stimulus onset. Each epoch was baseline corrected 351 from $-100 \mathrm{~ms}$ to $0 \mathrm{~ms}$. Epochs with amplitude values $\pm 150 \mu \mathrm{V}$ were excluded. For 352 consistency between systems, any epoch that was removed in one system was also 353 removed in the other. Thus, only those epochs accepted in both systems were 354 analysed. We then averaged accepted epochs to create ERP waveforms for both 355 standard and deviant tones. We calculated each participants' MMN by subtracting their 356 standard waveform from their deviant waveform (Luck, 2014). The MMN peak for each 357 EEG system was calculated as the average waveform value over the pre-determined 358 time window (https//osf.io/b764p) of $100 \mathrm{~ms}$ to $200 \mathrm{~ms}$ following stimulus onset. P300 processing. P300 EEG data were processed identically to MMN data with respect to ICA artefact removal, epoching and baseline removal, epoch rejection, and the creation of ERP waveforms for standard and deviant tones. However, the P300 waveform was the response to deviant tones only (i.e., we did not derive a component by subtracting waveforms as in the MMN). We calculated the P300 peak for each EEG system as the average waveform value over the pre-determined time window (https//osf.io/b764p) of $280 \mathrm{~ms}$ to $380 \mathrm{~ms}$ following stimulus onset. similarly to MMN and P300 data with respect to ICA artefact removal, epoching and baseline removal, epoch rejection. For each participant we created four waveforms: a) Upright faces; b) Inverted faces; c) Upright watches; and d) Inverted watches. The N170 370 peak was calculated as the average waveform value over the pre-determined time 371 window (https//osf.io/b764p) of $120 \mathrm{~ms}$ to $220 \mathrm{~ms}$. 
373 performed a spectral analysis with Fieldtrip using a single-taper fast Fourier transform

374 and a five-second, non-overlapping Hanning window in $0.2 \mathrm{~Hz}$ steps ('ft_freqanalysis'

375 function with length $=$ ' 5 ', method = ' $\mathrm{mtmfft}$ ', and taper $=$ 'hanning' arguments). Using

376 frequency-domain data, we calculated signal-to-noise ratio (SNR) values at each

377 frequency bin. SNR was calculated as the ratio of the amplitude at each frequency to

378 the average of the 20 surrounding frequency (10 on each side, excluding the

379 immediately adjacent bin; as performed in Liu-shuang, Norcia, \& Rossion, 2014). We

380 also calculated group z-scores for SNR values along the frequency spectrum (Liu-

381 shuang, Norcia, \& Rossion, 2014; Rossion, Prieto, Boremanse, Kuefner, \& Van Belle, 382 2012).

Resting state processing. After filtering, downsampling, and re-referencing, we

384 trimmed the data into eyes open and eyes closed trials. We then performed two spectral 385 analyses for each participant on each trial type and for each system using five-second, 386 non-overlapping Hanning window in $0.2 \mathrm{~Hz}$ steps in Fieldtrip ('ft_freqanalysis' function with length $=$ ' 5 ', method = 'mtmfft', and taper = 'hanning' arguments). One analysis was conducted on frequencies from 1 to $30 \mathrm{~Hz}$ in $1 \mathrm{~Hz}$ bins. The other analysis was conducted on alpha band $(8-12 \mathrm{~Hz})$ frequencies only, in increments of $0.004 \mathrm{~Hz}$ bins.

Group grand average power was calculated using 'ft_freqgrandaverage' and alpha band 391 power scalp topography maps were created with Fieldtrip using 'ft_topoplotER'.

\section{Analysis}


395 4.2; Morey \& Rouder, 2018) packages. To test our main question of whether the two 396 systems captured equivalent ERPs, we conducted Bayesian t-tests on specific channels 397 for each task. We did this because we employed a Bayesian stopping rule and required 398 a single statistical test for each task.

MMN analyses. We conducted a Bayesian t-test on the MMN components 400 measured by each system at Fz. We chose Fz for two reasons: 1) The MMN is most 401 robust in fronto-central regions (Garrido et al., 2009; Näätänen et al., 2007); and 2) We 402 wished to test the unique capability of Flex, relative to other Emotiv EEG systems, for 403 acquiring measurements along central locations.

404

To characterise the degree of similarity in waveforms captured by the two 405 systems, we calculated intra-class correlations (ICC) between waveforms (Badcock et 406 al., 2015; Badcock et al., 2013; Cassidy, Robertson, \& O'Connell, 2012; de Lissa et al., 407 2015; McArthur, Atkinson, \& Ellis, 2009; McArthur, Atkinson, \& Ellis, 2010). Between408 system ICCs were calculated for both standard and deviant tone waveform as well as 409 for MMN waveforms. ICCs were deemed significant if the $95 \%$ confidence intervals did 410 not include zero.

We also calculated measures of signal quality. The first of these measures was

412 the number of rejected epochs (i.e., number of epochs with amplitude values $\pm 150 \mu \mathrm{V}$ )

413 where a higher number of rejected epochs indicated poorer signal quality. We also 414 calculated the signal-to-noise ratio (SNR) averaged across all 16 electrodes of each 415 system. SNR was calculated as the root mean square of the signal (average amplitude 416 between 100 and $200 \mathrm{~ms}$ ) divided by the standard deviation of the baseline (-100 to 0 417 ms ; Maidhof, Rieger, Prinz, \& Koelsch, 2009; Marco-Pallares, Cucurell, Münte, Strien, 
418 \& Rodriguez-Fornells, 2011). To compare the signal qualities between systems, we 419 conducted a Bayesian t-test on the SNR values.

P300 analyses. We conducted a Bayesian t-test on the P300 peaks measured

421 by each system at Pz. We chose Pz for two reasons: 1) The P300 ERP is most robust 422 in parietal regions (Luck, 2014); and 2) We wished to test the unique capability of Flex, 423 relative to other Emotiv EEG systems, for acquiring measurements along central 424 locations. As with the MMN, we also calculated ICCs, the number of rejected epochs, 425 and SNRs. We calculated P300 SNRs as the root mean square of the signal (average 426 amplitude between 280 and $380 \mathrm{~ms}$ ) divided by the standard deviation of the baseline (427100 to 0 ms ; Maidhof, Rieger, Prinz, \& Koelsch, 2009; Marco-Pallares, Cucurell, Münte, 428 Strien, \& Rodriguez-Fornells, 2011).

N170 analyses. We conducted a Bayesian t-test on the N170 peak at P8 in the 430 upright faces condition. We chose P8 because the N170 is most robust over 431 occipitotemporal regions, particularly over the right hemisphere (Eimer, 2012; Luck, 432 2014). Additionally, we calculated values for P7 and conducted a Bayesian t-test, 433 though this analysis did not influence our pre-registered Bayesian stopping rule 434 (https://osf.io/b764p). As with the MMN and P300, we also calculated ICCs, the number 435 of rejected epochs, and SNRs. SNRs were calculated as the root mean square of the 436 signal (120 to $220 \mathrm{~ms}$ ) divided by the standard deviation of the baseline (-100 to $0 \mathrm{~ms}$;

437 Maidhof, Rieger, Prinz, \& Koelsch, 2009; Marco-Pallares, Cucurell, Münte, Strien, \& 438 Rodriguez-Fornells, 2011).

439 We also compared the timing of the N170 peak for each participant by indexing 440 the latency at which the minimum amplitude occurred during the time window of interest 
441 (i.e., $120 \mathrm{~ms}$ to $220 \mathrm{~ms}$ ). This allowed us to compare not only latency of the N170

442 response between the systems, but also between stimulus conditions (i.e., face vs.

443 watch and upright vs. inverted) as N170 latency is expected to be slightly, though

444 robustly, delayed when faces are inverted relative to when they are upright (Eimer, 445 2000).

446 SSVEP analyses. We conducted a Bayesian t-test on the SNR, averaged across 447 occipital $(\mathrm{O} 1, \mathrm{Oz}, \mathrm{O} 2)$ electrodes, at the stimulus frequency. We averaged the occipital 448 electrodes because SSVEP generally originates from the visual cortex and is thus most 449 pronounced over medial occipital areas (Norcia et al., 2015). We also calculated z450 scores on the SNR spectra for each system at each frequency bin. To determine 451 significance of SNR at each bin, we placed a Z-score threshold at $1.96(p<.05$; Norcia 452 et al., 2015).

$453 \quad$ Resting state analyses. We compared alpha power between the two systems 454 by averaging alpha across occipital sites $(\mathrm{O} 1, \mathrm{Oz}, \mathrm{O} 2)$. We then performed a Bayesian 455 ANOVA on alpha power with system (Neuroscan vs. Flex) and condition (eyes open vs. 456 eyes closed) as factors.

\section{Results}

458 MMN results.

459 Signal quality. For the MMN, one participant exceeded our threshold of $50 \%$ 460 rejected epochs (333) and was removed from further analysis and replaced with a new 461 participant. Table 3 displays descriptive statistics for epochs removed and SNRs. The

462 Bayesian t-test between MMN SNRs suggested insufficient evidence to determine 463 whether the systems differed. The number of rejected epochs was positively skewed so 
464 we used Wilcoxon Signed Rank Tests to compare the data between the two systems.

465 Overall, there was a statistically greater number of Flex epochs rejected in both tasks.

466 Generally, the numbers of rejected epochs were low and there were sufficient data with

467 which to conduct analyses.

Waveform similarity. Figure 3 depicts a comparison between the grand-average

waveforms of each system according to listening condition (passive vs. active) and tone

470 type (standard vs. deviant). ICC values can be found in their respective panels of

471 Figure 3. The waveforms used to create the MMN components (passive listening; $A$ and

472 C) were all very similar with the ICCs greater than 0.80 . We also calculated ICCs on the

473 MMN component waveforms (Figure 4). The waveforms were similar with an ICC of

4740.82 and $95 \%$ confidence interval of 0.80 to 0.84 . As none of the $95 \%$ confidence

475 intervals included zero, we considered all ICCs to be significant.

$476 \quad$ MMN component peak. Figure 4 contrasts the MMN component waveforms

477 between the two systems at Fz. We conducted a Bayesian t-test between the MMN

478 magnitudes of each system (MMN magnitude was calculated as the average signal

479 between 100 and $200 \mathrm{~ms}$ ) to determine whether there was a difference between the

480 components. Results favoured the null hypothesis with substantial evidence suggesting

481 no difference between the MMNs captured by the two systems, $\mathrm{BF}_{10}=0.25$.

482

We also conducted a post-hoc analysis using a new time window that better

483 represented the morphology of the MMN components observed in this study. To

484 calculate the time window of measurement, we first calculated the time point of the

485 minimum value of the overall grand mean MMN waveform between 0 and $200 \mathrm{~ms}$. We

486 then centred a $100 \mathrm{~ms}$ interval on this figure and calculated MMN values. The time point 
487 of the minimum of the overall MMN waveform was $116 \mathrm{~ms}$ and thus the new time 488 window of interest was 66 to $166 \mathrm{~ms}$.

After calculating MMN peak values for each participant, we conducted Bayesian t-test between the systems. These results provided substantial evidence that there was 491 no difference between the MMN peaks measured by the two systems, $\mathrm{BF}_{10}=0.23$. See

492 Table 4 for descriptive statistics of MMN peaks using both the pre-registered and post493 hoc calculations.

Table 5 shows the SNR of the MMN measured at Fz for each system. To 495 calculate the signal, both the pre-registered and post-hoc methods were used. Figure 5 496 shows the MMN topographic distributions for Neuroscan (A) and Flex (B). Topographic values were calculated using the post-hoc time window only. A Pearson correlation 498 suggested a moderate correlation of channel amplitudes between systems $(r=0.61, p<$ $499.001)$

500

501 502 503 504 505 506 507 508 509

\section{P300 results}

Signal quality. For the P300 paradigm, there was very strong evidence that Neuroscan SNR was larger than Flex SNR (6.60 vs 5.10; Table 3). In addition, there was a statistically greater number of Flex epochs rejected. These results suggest that, overall, the signal quality of Neuroscan was better than Flex in the P300 paradigm. Waveform similarity. The P300 waveforms were the responses to deviant tones in the active listening condition. Figure $3(\mathrm{D})$ and Figure $3(\mathrm{H})$ show P300 waveforms for Fz and Pz, respectively. The waveforms of both systems were very similar (ICCs > 0.93). They did not include zero in their $95 \%$ confidence intervals and so were considered significant. 
511 measured at Pz. We conducted a Bayesian t-test between P300 peak values of each

512 system. Results indicated substantial evidence that there was no difference between

513 the P300s captured by the two systems, $\mathrm{BF}_{10}=0.26$.

514 For consistency between P300 and MMN analyses, we calculated a post-hoc

515 time window by calculating the maximum value of the overall grand mean ERP between

516200 and $400 \mathrm{~ms}$. We centred a new time window of $100 \mathrm{~ms}$ on the timepoint at which

517 this maximum value occurred. Our new time window was calculated as $198-298 \mathrm{~ms}$.

518 After calculating P300 values for each participant based on this window, we conducted

519 another Bayesian t-test between the systems. These results mirrored our initial analysis

520 and suggested that there was no difference between the P300 peaks measured by the

521 two systems, $\mathrm{BF}_{10}=0.32$. See Table 4 for descriptive statistics.

522 P300 SNR values for each system are presented in Table 5. Values reflecting

523 both the pre-registered and post-hoc time windows are shown. Figure 5 displays the

524 topographic distributions of $\mathrm{P} 300$ peaks at $\mathrm{Pz}$ for Neuroscan (C) and Flex (D). As with

525 the MMN, we observed a moderate correlation of channel values between systems $(r=$

$5260.60, p<.001)$

527 N170 results

528 Signal Quality. For the N170, there was substantial evidence that the

529 Neuroscan SNR of was higher than Flex (6.04 vs 5.12; Table 3). However, there was no

530 difference in the number of rejected epochs between the two systems.

532 waveforms over the left (P7) and right (P8) hemispheres for each stimulus type with 
533 ICCs found in their respective panels. As none of the $95 \%$ confidence intervals included

534 zero, we considered all ICCs to be significant. Right hemisphere waveforms (P8)

535 exhibited a higher degree of similarity than left hemisphere waveforms (P7).

536 The N170 peak. We performed a Bayesian t-test between the N170 peaks in the 537 upright face condition at channel P8 (Figure 6, Panel B). Results indicated very strong 538 evidence that the $\mathrm{N} 170$ peaks measured by the two systems were different, $\mathrm{BF}_{10}=$ 539 35.88. We also performed a Bayesian t-test between the N170 peaks in the upright face

540 condition at channel P7 (Figure 6, Panel A). Results also indicated strong evidence that 541 the $\mathrm{N} 170$ peaks measured by the two systems were different, $\mathrm{BF}_{10}=22.59$. Table 6 for 542 descriptive statistics.

543 SNR values for each of the N170 stimulus conditions at P7 and P8 are displayed

544 in Table 5 and Figure 7 displays the topographic distributions of N170 peaks across the 545 stimulus conditions. Again, topographies of the two systems were moderately correlated $546(r=0.56, p<.001)$.

\section{SSVEP results}

$548 \quad$ Figure 8 shows the SNR values for each system across the frequency spectrum

$549(1-30 \mathrm{~Hz})$. The Bayesian t-test at the stimulus frequency provided decisive evidence 550 that the magnitudes of the system SNRs differed, $B_{10}=51764$. The SNR z-scores for 551 both systems exceeded 1.96 at the stimulus frequency (Table 7 ). This indicated that, 552 although the Neuroscan SSVEP response was larger than the Flex response, both 553 systems were still capable of registering an SSVEP signature. 


\section{Resting state results}

$555 \quad$ Figure 9 depicts the topographical alpha power $(8-12 \mathrm{~Hz})$ distribution for eyes

556 open and eyes closed condition for each system. Table 8 provides descriptive statistics

557 for alpha power and the suppression measure. Figure 10 contrasts power for each

558 system and condition across the $1-30 \mathrm{~Hz}$ frequency spectrum. The Bayesian ANOVA

559 indicated very strong evidence for a main effect of condition $\left(B_{10}=32.27\right)$ and

560 substantial evidence for no effect of system $\left(B_{10}=0.28\right)$. Thus, both Neuroscan and

561 Flex measured similar differences in alpha power between eyes open and eyes closed 562 conditions (see Table 8).

563

\section{Discussion}

564

The aim of the current study was to validate a consumer-grade EEG system,

565 Emotiv EPOC Flex. To achieve this, we simultaneously recorded EEG with Flex and

566 Neuroscan across an array of tasks designed to provide comparisons in temporal and

567 phasic domains. In the time domain we measured auditory and visual ERPs and in the

568 frequency domain we measured SNR response to a flickering screen and alpha power 569 during a resting state task.

570 Our first main finding was that Flex recorded auditory ERPs similar to those

571 measured by Neuroscan. This was evident in the finding that the magnitude of MMN

572 and P300 peaks were equivalent between systems. Further, ERP waveforms in both the

573 passive and active listening conditions were very similar between systems as were the

574 scalp topographies. The similarity of signal distribution across all electrodes is

575 particularly noteworthy as it suggests that even sites that were distant from the main

576 sources of the ERP-relevant signals still show similar measurements between the two 
577 systems. Comparison of the face-sensitive N170 waveforms captured by the two

578 systems also showed a high degree of similarity, particularly over the right hemisphere.

579 Again, scalp topographies demonstrated a high degree of signal similarity, even at sites

580 distant from occipito-temporal areas. Although we observed similarity between face-

581 related signals we did find substantial evidence that the actual magnitudes of the N170

582 peaks were different between systems. Although this finding indicates that the

583 amplitude of the N170 effect measured by Flex and Neuroscan was different, it does not

584 mean that one of the systems was incapable of measuring an N170 ERP. In fact, Flex

585 actually measured a larger-amplitude N170 peak. This was likely due to the constraints

586 of a simultaneous setup wherein system sensors could not be situated in the exact

587 same locations. As it was the system being validated, we placed Flex sensors in the

588 precise location of interest (P8 in this case) and Neuroscan sensors immediately

589 adjacent along an axis towards Cz. This likely positioned Flex in an advantageous

590 position for capturing face-related neural signals and contributed to the larger N170

591 peak at P8. Indeed, a recent study examining localisation of face-related brain

592 signatures found the largest N170 effect at PO10 (Gao, Conte, Richards, Xie, \&

593 Hanayik, 2019). Thus, in the current study, the Flex sensor was in closer proximity to

594 PO10 than the Neuroscan sensor. A similar pattern and explanation has been noted in 595 an N170 validation of another Emotiv system (Emotiv EPOC; de Lissa et al., 2015).

596 Another potential explanation for the larger amplitude N170 peak observed for

597 Flex may be the fact that it exhibited a smaller P1 value relative to Neuroscan (see

598 Figure 6). This highlights the sensitivity of these types of comparisons to the calculation

599 method with which ERP component and peak values are derived. A "fairer" comparison 
600 may have been a so-called peak-to-trough comparison in which we calculated N170

601 values based on the difference between P1 and N1 values. Irrespective of the

602 calculation method, it is the implicit aim of validation studies such as the current one to

603 determine whether the system being validated collects accurate data, not whether it

604 collects data identical to that of another system. Considering the high degree of

605 morphological similarity between N170 waveforms, it is apparent that Flex is indeed

606 capable of indexing visual ERPs.

607 Our findings also demonstrate that Flex measures acceptable spectral EEG data.

608 This is evidenced by results showing higher alpha power during eyes closed relative to

609 and eyes open conditions. Further, we observed no differences in alpha power between

610 Flex and Neuroscan. We did, however, observe a large difference between the systems

611 in the SNR measured in an SSVEP task. The Neuroscan SNR was orders of magnitude

612 larger than Flex SNR. Why this would be the case is not immediately evident. However,

613 we speculate that the lower recording resolution of Flex-129 Hz vs $1000 \mathrm{~Hz}$ for

614 Neuroscan —may have introduced temporal smearing wherein in the EEG response at

615 the stimulus frequency was binned to adjacent frequencies thereby lowering SNR

616 values. Indeed, even minute variations in system timing can degrade evoked EEG

617 signatures (see Hairston, 2012; Ouyang, Sommer, \& Zhou, 2016) and, given their often-

618 high frequency stimulation rates, SSVEPs are particularly susceptible. Though Flex

619 measured smaller SNR values compared to Neuroscan, the SNR peaks observed at the

620 stimulus frequency were nonetheless significant compared to values at surrounding

621 frequencies. 
622 Another finding was that overall signal quality was lower for Flex than for

623 Neuroscan in the P300 task. While we did not have enough evidence for SNR

624 differences in the MMN task to make a conclusion, the number of threshold-rejected

625 epochs in each of the auditory tasks was higher for Flex than for Neuroscan. The N170

626 SNR was also greater for Neuroscan than Flex. However, the number of rejected

627 epochs was not statistically different. Overall, the differences in SNR were not surprising

628 as Neuroscan has a much higher resolution than Flex (24-bit vs. 14-bit, respectively)

629 and would thus be expected to be more sensitive to signal fluctuation. This would be

630 particularly true when calculating SNR over the entire scalp where much of the

631 measurement is distant from the signal generator of interest and thus more susceptible

632 to noise-distortion. The reason for disparity between the two modalities was likely due

633 to the fact that participants were given explicit opportunity to blink their eyes (during

634 'blink' screens) while performing the N170 task, which would have placed most motion

635 artefacts outside of epoch limits. Explicit opportunities to move or blink were not present

636 during the auditory tasks. As such, there was likely more motion artefacts present in

637 these epochs and Flex was more sensitive to these. However, considering that the

638 median number of rejected artefacts represented approximately $1 \%$ of trials in both

639 passive and active conditions, we did not consider signal quality to be an issue with

640 Flex.

641 One limitation of this study is the nature of the simultaneous setup of the two

642 EEG systems. Because we wished to test whether the signals measured by the two

643 systems were similar, we placed the respective electrodes immediately adjacent to each

644 other at each site. While this allowed us to assess the similarity of signals, it also 
645 created a situation in which we could not guarantee that there was no low-impedance

646 electrical bridging between the electrodes of the two systems. In other words, it was

647 possible that the signals we measured with Flex were not the same as if Flex had been

648 used alone. Unfortunately, to mitigate this limitation required either a between-subjects

649 design (one system per participant) or to do two separate setups with each participant.

650 The downside to the first solution is that there is a loss of statistical power to detect

651 differences between the systems. The downside to the second solution is the potential

652 for differences in intra-individual participant characteristics from system to system (e.g.,

653 fatigue, motivation). Therefore, we deemed the advantages of the dual setup

654 methodology greater than disadvantages.

655 Another potential limitation is the fact that is not possible to quantify the exact 656 impedance value of Flex. The Emotiv software uses a "traffic light" impedance feedback 657 system wherein users adjust electrodes until an indicator light turns green, which 658 signifies impedance of less than $20 \mathrm{k} \Omega$. This may concern some researchers as many 659 protocols aim for impedances of less than $5 \mathrm{k} \Omega$. However, the less-than- $5 \mathrm{k} \Omega$ 660 convention is typical of "passive" EEG systems that use a ground circuit to subtract 661 common mode voltage and achieve clean EEG recordings (Luck, 2014). Flex is an 662 "active" EEG system that uses a common mode sense and driven right leg to optimise 663 EEG signal. Although explanation of the technical aspects of passive and active 664 systems is outside the scope of this paper, we note that because of their circuitry, 665 impedance values are less of a concern with active systems (Keil et al., 2014). 666 Additionally, work by Kappenman and Luck (2010) suggests that while high-impedance 
667 setups may result in lower signal-to-noise ratio, this can be attenuated with higher trial 668 counts, filtering, and artifact rejection.

669 In summary, these results support Flex is a valid device for EEG and ERP

670 research. Naturally, there are compromises when choosing a lower-cost, consumer-

671 grade EEG devices over more expensive research-grade devices. Both the lower bit

672 rate and the lower sampling rate of Flex mean that it is not as sensitive as Neuroscan to

673 small variations in EEG signals. This can also mean that these devices are 'noisier' than

674 research-grade devices. However, these are shortcomings that are typical to all devices

675 in this class and, for some researchers, are offset by their affordability and flexibility.

676

\section{Conclusions}

677 Overall, our findings suggest that Flex is capable of collecting research-quality

678 data. It can register both event-related and spectral EEG signatures similar to those of a

679 research-grade system. The validity of the Flex system makes it a suitable alternative to

680 research-grade systems; an alternative that may be appealing to some researchers for

681 whom traditional lab-based EEG systems are cost prohibitive. Additionally, the easy, 682 convenient setup makes Flex suitable for use with atypical populations (e.g., children

683 and sensitive individuals) by ameliorating participant discomfort experienced from long 684 setup times and the use of electroconductive gel. Finally, the portability of Flex allows it 685 to be used outside of the laboratory and in more naturalistic settings. By mitigating 686 financial, methodological, and practical barriers, Flex may facilitate neuroscience 687 research on topics and in locations in which it would otherwise not be feasible. 
691 Paul Sowman who provided support with the SSVEP analysis as well as Peter de Lissa 692 who provided the stimulus for the N170 paradigm. Finally, we would like to thank the 693 participants who volunteered their time.

694

\section{Conflict of Interest Statement}

695 This work was made possible by an industry partnership grant between Emotiv 696 Pty. Ltd. and Macquarie University. Emotiv contributions to this project included

697 hardware, software, technical support, and contribution to the first author's salary by 698 way of a cofounded fellowship. Emotiv had no influence on data collection, data 699 analyses, or decision to publish this project (see the pre-registration available at 700 https://osf.io/b764p).

701 
703 Alamdari, N., Haider, A., Arefin, R., Verma, A. K., Tavakolian, K., \& Fazel-Rezai, R.

704 (2016). A review of methods and applications of brain computer interface systems.

705 IEEE International Conference on Electro Information Technology, 2016-Augus,

$706 \quad 345-350$. https://doi.org/10.1109/EIT.2016.7535263

707 Amzica, F., \& Steriade, M. (1998). Electrophysiological correlates of sleep delta waves.

708 Electroencephalography and Clinical Neurophysiology, 107(2), 69-83.

709 https://doi.org/10.1016/S0013-4694(98)00051-0

710 Badcock, N. A., Mousikou, P., Mahajan, Y., de Lissa, P., Thie, J., \& McArthur, G.

711 (2013). Validation of the Emotiv EPOC ${ }^{\circledR}$ EEG gaming system for measuring

712 research quality auditory ERPs. PeerJ, 1, e38. https://doi.org/10.7717/peerj.38

713 Badcock, N. A., Preece, K. A., de Wit, B., Glenn, K., Fieder, N., Thie, J., \& McArthur, G.

714 (2015). Validation of the Emotiv EPOC EEG system for research quality auditory

715 event-related potentials in children.

716 https://doi.org/http://dx.doi.org/10.7717/peerj.907

717 Barham, M. P., Clark, G. M., Hayden, M. J., Enticott, P. G., Conduit, R., \& Lum, J. A. G.

718 (2017). Acquiring research-grade ERPs on a shoestring budget: A comparison of a 719 modified Emotiv and commercial SynAmps EEG system. Psychophysiology, 54(9),

720 1393-1404. https://doi.org/http://dx.doi.org/10.1111/psyp.12888

721 Barry, R. J., \& De Blasio, F. M. (2017). EEG differences between eyes-closed and eyes722 open resting remain in healthy ageing. Biological Psychology, 129(April), 293-304.

723 https://doi.org/10.1016/j.biopsycho.2017.09.010

724 Billeci, L., Sicca, F., Maharatna, K., Apicella, F., Narzisi, A., Campatelli, G., ... Muratori, 
725 F. (2013). On the application of Quantitative EEG for characterizing autistic brain: A

726 systematic review. Frontiers in Human Neuroscience, 7(JUL), 1-15.

727 https://doi.org/10.3389/fnhum.2013.00442

728 Bonnefond, M., \& Jensen, O. (2012). Alpha oscillations serve to protect working

729 memory maintenance against anticipated distracters. Current Biology, 22(20),

730 1969-1974. https://doi.org/10.1016/j.cub.2012.08.029

731 Brainard, D. H. (1997). The Psychophysics Toolbox. Spatial Vision, 10(4), 433-436.

732 https://doi.org/10.1163/156856897X00357

733 Cassidy, S. M., Robertson, I. H., \& O'Connell, R. G. (2012). Retest reliability of event-

734 related potentials: Evidence from a variety of paradigms. Psychophysiology, 49(5),

735 659-664. https://doi.org/10.1111/j.1469-8986.2011.01349.x

736 de Lissa, P., Sörensen, S., Badcock, N., Thie, J., \& Mcarthur, G. (2015). Measuring the

737 face-sensitive N170 with a gaming EEG system: A validation study. Journal of

$738 \quad$ Neuroscience Methods, 253, 47-54.

739 https://doi.org/10.1016/j.jneumeth.2015.05.025

740 De Vos, M., Kroesen, M., Emkes, R., \& Debener, S. (2014). P300 speller BCI with a 741 mobile EEG system: comparison to a traditional amplifier. Journal of neural

742 engineering, 11(3), 036008. https://doi.org/10.1088/1741-2560/11/3/036008

743 Debener, S., Beauducel, A., Nessler, D., Brocke, B., Heilemann, H., \& Kayser, J.

744 (2000). Is resting anterior EEG alpha asymmetry a trait marker for depression?

745 Findings for healthy adults and clinically depressed patients. Neuropsychobiology,

$746 \quad 41(1), 31-37$. https://doi.org/10.1159/000026630 
747 Debener, S., Minow, F., Emkes, R., Gandras, K., \& De Vos, M. (2012). How about

748 taking a low-cost, small, and wireless EEG for a

749

750 walk?. Psychophysiology, 49(11), 1617-1621. https://doi.org/10.1111/j.14698986.2012.01471.x

751 Delorme, A., \& Makeig, S. (2004). EEGLAB: an open source toolbaox for anlaysis of 752 single-trial EEG dynamics including independent component anlaysis. Journal of $753 \quad$ Neuroscience Methods, 134, 9-21. https://doi.org/10.1016/j.jneumeth.2003.10.009

754 Duvinage, M., Castermans, T., Petieau, M., Hoellinger, T., Cheron, G., \& Dutoit, T. 755 (2013). Performance of the Emotiv Epoc headset for P300-based applications.

756 757 BioMedical Engineering Online, 12(1), 1-15. https://doi.org/10.1186/1475-925X-12-

758 Düzel, E., Penny, W. D., \& Burgess, N. (2010). Brain oscillations and memory. Current $759 \quad$ Opinion in Neurobiology, 20(2), 143-149.

$760 \quad$ https://doi.org/10.1016/j.conb.2010.01.004

761 Eimer, M. (2000). Effects of face inversion on the structural encoding and recognition of 762 faces. Cognitive Brain Research, 10(1-2), 145-158. https://doi.org/10.1016/s0926763 6410(00)00038-0

764 Eimer, M. (2012). The Face-Sensitive N170 Component of the Event-Related Brain 765 Potential. Oxford Handbook of Face Perception, 329-344. https://doi.org/10.1093/oxfordhb/9780199559053.013.0017

767 Feuerriegel, D., Churches, O., Hofmann, J., \& Keage, H. A. D. (2015). The N170 and 768 face perception in psychiatric and neurological disorders: A systematic review. $769 \quad$ Clinical Neurophysiology, 126(6), 1141-1158. 
771 Gao, C., Conte, S., Richards, J. E., Xie, W., \& Hanayik, T. (2019). The neural sources of

772 N170: Understanding timing of activation in face-selective areas.

773 Psychophysiology, 56(6), e13336.

774 Garrido, M. I., Kilner, J. M., Stephan, K. E., \& Friston, K. J. (2009). The mismatch

775 negativity: A review of underlying mechanisms. Clinical Neurophysiology, 120(3),

776 453-463. https://doi.org/10.1016/j.clinph.2008.11.029

777 Hairston, W. D. (2012). Accounting for Timing Drift and Variability in Contemporary

778 Electroencepholography ( EEG ) Systems. DTIC Document, (March), 1-22.

$779 \quad$ Retrieved from

780 http://oai.dtic.mil/oai/oai?verb=getRecord\&metadataPrefix=html\&identifier=ADA561

$781 \quad 715$

782 Hinojosa, J. A., Mercado, F., \& Carretié, L. (2015). N170 sensitivity to facial expression:

783 A meta-analysis. Neuroscience and Biobehavioral Reviews, 55, 498-509.

784 https://doi.org/10.1016/j.neubiorev.2015.06.002

785 Itier, R. J., Latinus, M., \& Taylor, M. J. (2006). Face, eye and object early processing:

786 What is the face specificity? Neurolmage, 29(2), 667-676.

$787 \quad$ https://doi.org/10.1016/j.neuroimage.2005.07.041

788 Itier, R. J., \& Taylor, M. J. (2004). N170 or N1? Spatiotemporal Differences between

789 Object and Face Processing Using ERPs. Cerebral Cortex, 14(2), 132-142.

$790 \quad$ https://doi.org/10.1093/cercor/bhg111

791 Jacobs, G. D., \& Friedman, R. (2004). EEG spectral analysis of relaxation techniques.

$792 \quad$ Applied Psychophysiology Biofeedback, 29(4), 245-254. 
794 Jarosz, A. F., \& Wiley, J. (2014). Journal of Problem Solving What Are the Odds? A 795 Practical Guide to Computing and Reporting Bayes Factors. 7, 2-9.

796 Kappenman, E. S., \& Luck, S. J. (2010). The effects of electrode impedance on data 797 quality and statistical significance in ERP recordings. Psychophysiology, 47(5),

798 888-904. doi:10.1111/j.1469-8986.2010.01009.x.

799 Keil, A., Debener, S., Gratton, G., Junghöfer, M., Kappenman, E. S., Luck, S. J., ... \& 800 Yee, C. M. (2014). Committee report: publication guidelines and recommendations 801 for studies using electroencephalography and 802 magnetoencephalography. Psychophysiology, 51(1), 1-21. doi:

$803 \quad$ 10.1111/psyp.12147.

804 Kim, S. H., Grammer, J., Benrey, N., Morrison, F., \& Lord, C. (2018). Stimulus 805 processing and error monitoring in more-able kindergarteners with autism spectrum 806 disorder: a short review and a preliminary Event-Related Potentials study.

$807 \quad$ European Journal of Neuroscience, 47(6), 556-567.

808 https://doi.org/10.1111/ejn.13580

809 Kleiner, M., Brainard, D., Pelli, D., Ingling, A., Murray, R., \& Broussard, C. (2007).

810 What's new in psychtoolbox-3. Perception, 36(14), 1-16.

811 Klimesch, W. (1999). EEG alpha and theta oscillations reflect cognitive and memory 812 performance: A review and analysis. Brain Research Reviews, 29(2-3), 169-195. 813 https://doi.org/10.1016/S0165-0173(98)00056-3

814 Krigolson, O. E., Williams, C. C., Norton, A., Hassall, C. D., \& Colino, F. L. (2017).

815 Choosing MUSE: Validation of a low-cost, portable EEG system for ERP research. 
816 Frontiers in Neuroscience, 11(MAR), 1-10.

817 https://doi.org/10.3389/fnins.2017.00109

818 Liu-shuang, J., Norcia, A. M., \& Rossion, B. (2014). An objective index of individual face 819 discrimination in the right occipito-temporal cortex by means of fast periodic oddball 820 stimulation. Neuropsychologia, 52, 57-72.

821 https://doi.org/10.1016/j.neuropsychologia.2013.10.022

822 Lochy, A., Van Belle, G., \& Rossion, B. (2015). A robust index of lexical representation 823 in the left occipito-temporal cortex as evidenced by EEG responses to fast periodic 824 visual stimulation. Neuropsychologia, 66, 18-31.

825 https://doi.org/10.1016/j.neuropsychologia.2014.11.007

826 Luck, S. J. (1998). Neurophysiology of selective attention. Attention, 1.

827 Luck, S. J. (2014). An introduction to the event-related potential technique. MIT press.

828 Mahajan, Y., \& McArthur, G. (2010). Does combing the scalp reduce scalp electrode 829 impedances? Journal of Neuroscience Methods, 188(2), 287-289.

830 https://doi.org/10.1016/j.jneumeth.2010.02.024

831 McArthur, G. M., Atkinson, C., \& Ellis, D. (2009). Atypical brain responses to sounds in 832 children with specific language and reading impairments. Developmental Science, 833 12(5), 768-783. https://doi.org/10.1111/j.1467-7687.2008.00804.x

834 McArthur, G. M., Atkinson, C. M., \& Ellis, D. (2010). Can training normalize atypical 835 passive auditory ERPs in children with SRD or SLI? Developmental $836 \quad$ Neuropsychology, 35(6), 656-678. https://doi.org/10.1080/87565641.2010.508548

837 McMahon, C. M., Boisvert, I., de Lissa, P., Granger, L., Ibrahim, R., Lo, C. Y., ... 838 Graham, P. L. (2016). Monitoring alpha oscillations and pupil dilation across a 
performance-intensity function. Frontiers in Psychology, 7(MAY), 1-12.

840 https://doi.org/10.3389/fpsyg.2016.00745

841 Melnik, A., Legkov, P., Izdebski, K., Kärcher, S. M., Hairston, W. D., Ferris, D. P., \& König, P. (2017). Systems, Subjects, Sessions: To What Extent Do These Factors Influence EEG Data? Frontiers in Human Neuroscience, 11(March), 1-20.

844 https://doi.org/10.3389/fnhum.2017.00150

845

Morey, R. D., \& Rouder, J. N. (2018). BayesFactor. Retrieved from

846 https://richarddmorey.github.io/BayesFactor/

Morrison, C., Rabipour, S., Taler, V., Sheppard, C., \& Knoefel, F. (2019). Visual EventLiterature Review. Current Alzheimer Research, 16(1), 67-89.

Näätänen, R., Paavilainen, P., Rinne, T., \& Alho, K. (2007). The mismatch negativity

Norcia, A. M., Appelbaum, L. G., Ales, J. M., Cottereau, B. R., \& Rossion, B. (2015). https://doi.org/10.1167/15.6.4.doi

859 Nunez, P. L., \& Srinivasan, R. (2006). Electric fields of the brain: the neurophysics of 860 EEG. Oxford University Press, USA.

861 Oostenveld, R., Fries, P., Maris, E., \& Schoffelen, J. M. (2011). FieldTrip: Open source 
862

863

864

865

866

867

868

869

870

871

872

873

874

875

876

877

878

879

880

881

882

883

884

software for advanced analysis of MEG, EEG, and invasive electrophysiological data. Computational Intelligence and Neuroscience, 2011. https://doi.org/10.1155/2011/156869

Ouyang, G., Sommer, W., \& Zhou, C. (2016). Reconstructing ERP amplitude effects after compensating for trial-to-trial latency jitter: A solution based on a novel application of residue iteration decomposition. International Journal of Psychophysiology, 109, 9-20. https://doi.org/10.1016/j.jpsycho.2016.09.015

Pelli, D. G. (1997). The VideoToolbox software for visual psychophysics: Transforming numbers into movies. Spatial Vision, 10(4), 437-442. https://doi.org/10.1016/j.jmgm.2005.11.005

Peter, B., McCollum, H., Daliri, A., \& Panagiotides, H. (2019). Auditory gating in adults with dyslexia: An ERP account of diminished rapid neural adaptation. Clinical Neurophysiology, 130(11), 2182-2192. https://doi.org/10.1016/j.clinph.2019.07.028

Polich, J. (2007). Updating P300: An integrative theory of P3a and P3b. Clinical Neurophysiology, 118(10), 2128-2148. https://doi.org/10.1016/j.clinph.2007.04.019

Polich, J., \& Kok, A. (1995). Cognitive and biological determinants of P300: an integrative review. Biological Psychology, 41(2), 103-146. https://doi.org/10.1016/0301-0511(95)05130-9

Ratti, E., Waninger, S., Berka, C., Ruffini, G., \& Verma, A. (2017). Comparison of medical and consumer wireless EEG systems for use in clinical trials. Frontiers in Human Neuroscience, 11(August), 1-7. https://doi.org/10.3389/fnhum.2017.00398 Ray, W. J., \& Cole, H. W. (1985). EEG alpha activity reflects attentional demands, and beta activity reflects emotional and cognitive processes. Science, 228(4700), 750- 
Rossion, B., Gauthier, I., Tarr, M. J., Pierenne, D., Debatisse, D., \& Despland, P. A. to inverted objects: Electrophysiological evidence of face-specific processes in the human brain. Neurolmage, 9(6 PART II).

Rossion, B., Prieto, E. A., Boremanse, A., Kuefner, D., \& Van Belle, G. (2012). A steady-state visual evoked potential approach to individual face perception: Effect of inversion, contrast-reversal and temporal dynamics. Neurolmage, 63(3), 15851600. https://doi.org/10.1016/j.neuroimage.2012.08.033

Sauseng, P., Klimesch, W., Stadler, W., Schabus, M., Doppelmayr, M., Hanslmayr, S., 895 ... Birbaumer, N. (2005). A shift of visual spatial attention is selectively associated 896 with human EEG alpha activity. European Journal of Neuroscience, 22(11), 29172926. https://doi.org/10.1111/j.1460-9568.2005.04482.x

Schieber, F. (2018). Mex-File Plug-in for Fast MATLAB Port I/O. Retrieved from 899 http://apps.usd.edu/coglab/psyc770//O64.html

Schwartz, S., Shinn-Cunningham, B., \& Tager-Flusberg, H. (2018). Meta-analysis and 901

902

903 systematic review of the literature characterizing auditory mismatch negativity in individuals with autism. Neuroscience and Biobehavioral Reviews, 87(June 2017), 106-117. https://doi.org/10.1016/j.neubiorev.2018.01.008

Swords, G. M., Nguyen, L. T., Mudar, R. A., \& Llano, D. A. (2018). Auditory system dysfunction in Alzheimer disease and its prodromal states: A review. Ageing Research Reviews, 44(April), 49-59. https://doi.org/10.1016/j.arr.2018.04.001

07 Team, R. C. (2013). R: A language and environment for statistical computing. 
908 Uusberg, A., Uibo, H., Kreegipuu, K., \& Allik, J. (2013). EEG alpha and cortical inhibition 909 in affective attention. International Journal of Psychophysiology, 89(1), 26-36.

$910 \quad$ https://doi.org/10.1016/j.ijpsycho.2013.04.020

911 Vecchio, F., Babiloni, C., Lizio, R., De Vico Fallani, F., Blinowska, K., Verrienti, G., ... 912 Rossini, P. M. (2013). Resting state cortical EEG rhythms in Alzheimer's disease:

913 Toward EEG markers for clinical applications: A review. Supplements to Clinical $914 \quad$ Neurophysiology, 62, 223-236. https://doi.org/10.1016/B978-0-7020-5307-8.00015$915 \quad 6$

916 Vogel, E. K., \& Luck, S. J. (2002). Delayed working memory consolidation during the 917 attentional blink. Psychonomic Bulletin and Review, 9(4), 739-743.

$918 \quad$ https://doi.org/10.3758/BF03196329

919 Vogel, E. K., Luck, S. J., \& Shapiro, K. L. (1998). Electrophysiological Evidence for a 920 Postperceptual Locus of Suppression during the Attentional Blink. Journal of 921 Experimental Psychology: Human Perception and Performance, 24(6), 1656-1674. $922 \quad$ https://doi.org/10.1037/0096-1523.24.6.1656

923 Wickham, H., Averick, M., Bryan, J., Chang, W., McGowan, L., François, R., .. Yutani, 924 H. (2019). Welcome to the Tidyverse. Journal of Open Source Software, 4(43), $925 \quad$ 1686. https://doi.org/10.21105/joss.0168.

926 Williams, N., McArthur, G., \& Badcock, N. (2020). 10 years of EPOC: A scoping review 927 of Emotiv's portable EEG device. bioRxiv.

928 doi: https://doi.org/10.1101/2020.07.14.202085

929 
Figure 1

Flex electrode positions for the validation study.

Letters represent Flex sensor labels. Blue/red font represents left/right side sensors.

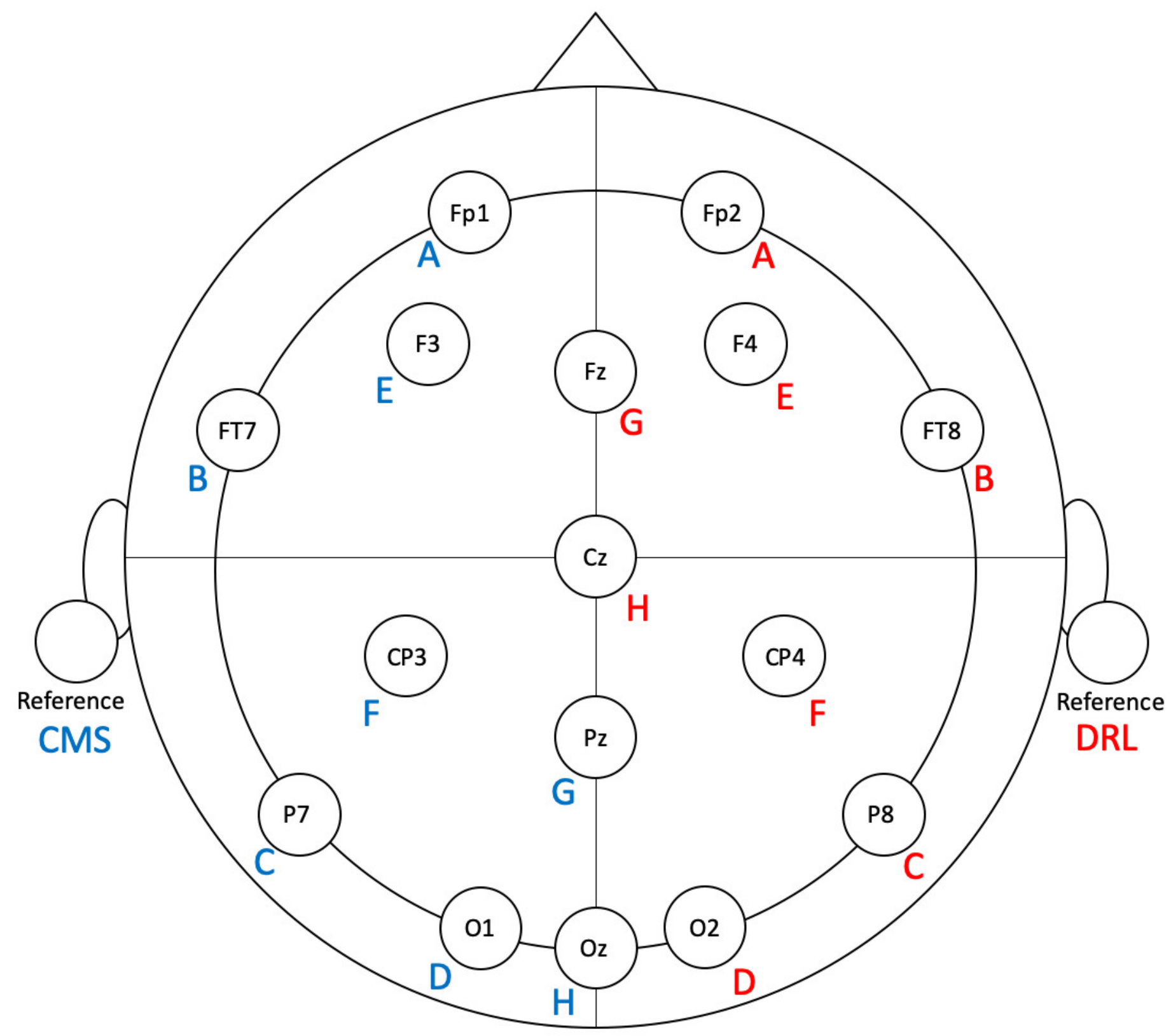


Figure 2

Flex validation setup schematic.

EEG data were collected simultaneously and acquired with Curry (Neuroscan) and Emotiv Pro (Flex) software. Event-marking was achieved using parallel port triggers.

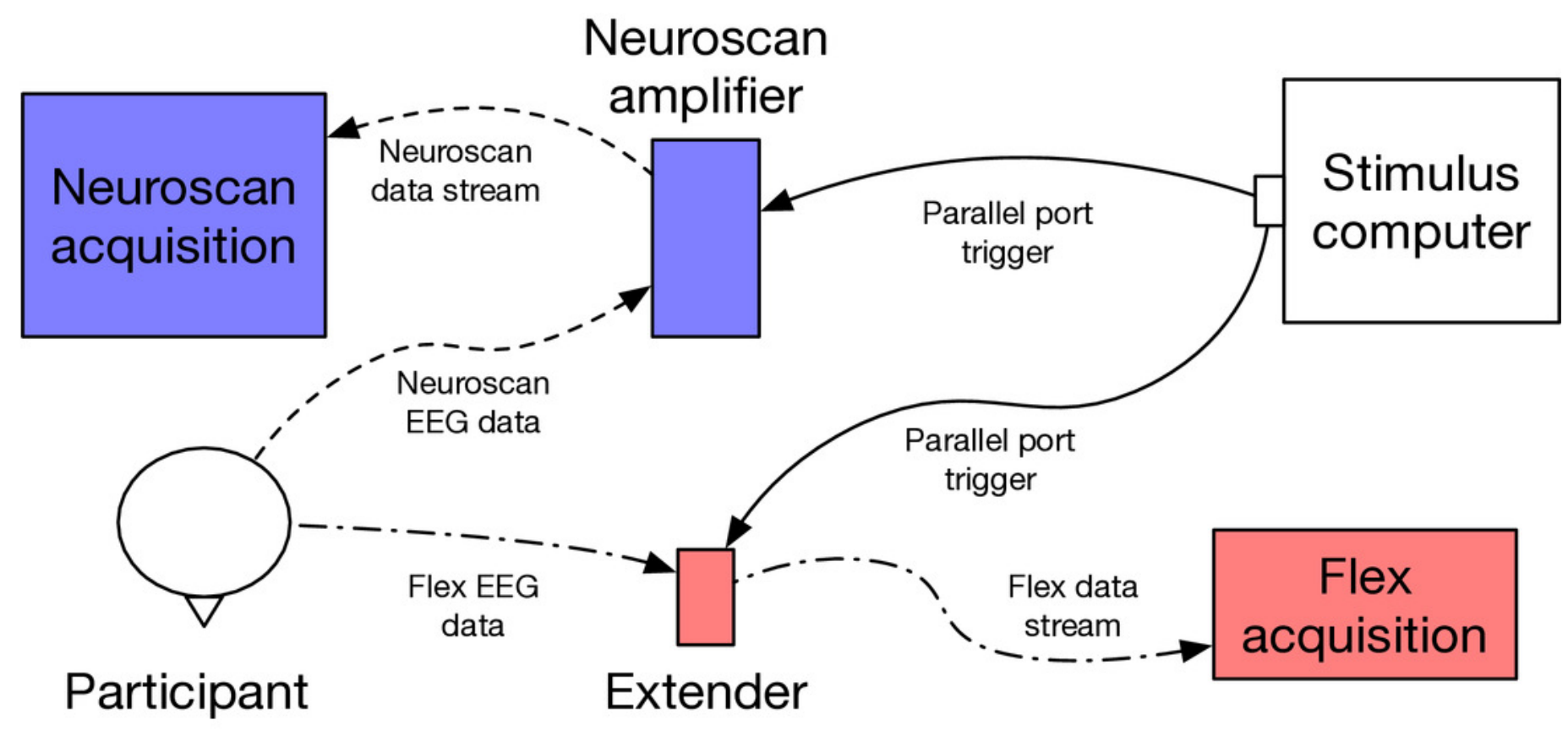




\section{Figure 3}

Group auditory ERP waveforms and intraclass correlations [95\% confidence interval] for Neuroscan and Flex systems by listening condition and tone type.

Passive waveforms (A, C, E, and G) were used to create the MMN waveform. Deviant waveforms in the active listening condition ( $D$ and $H$ ) were the P300 waveforms. (A) - (D) depict channel Fz. $(E)$ - (H) depict channel Pz. Intraclass correlations were considered significant if the $95 \%$ confidence interval did not include zero. 


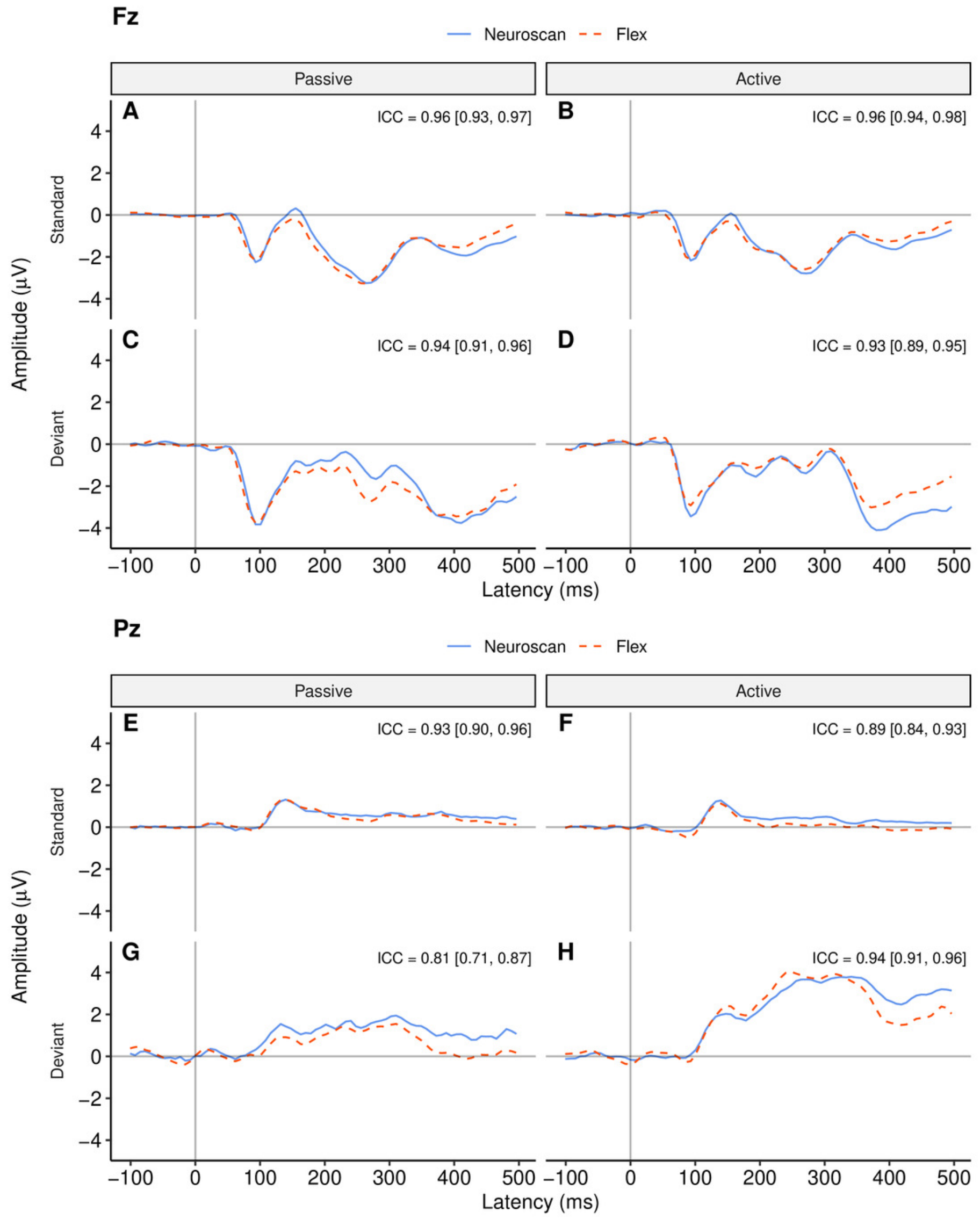


Figure 4

MMN component waveforms (deviant tones - standard tones) for each system measured at Fz.

ICCs between the two waveforms were considered significant if the $95 \%$ confidence interval did not include zero. The shaded areas denote the pre-registered time window of interest.

- Neuroscan - - Flex

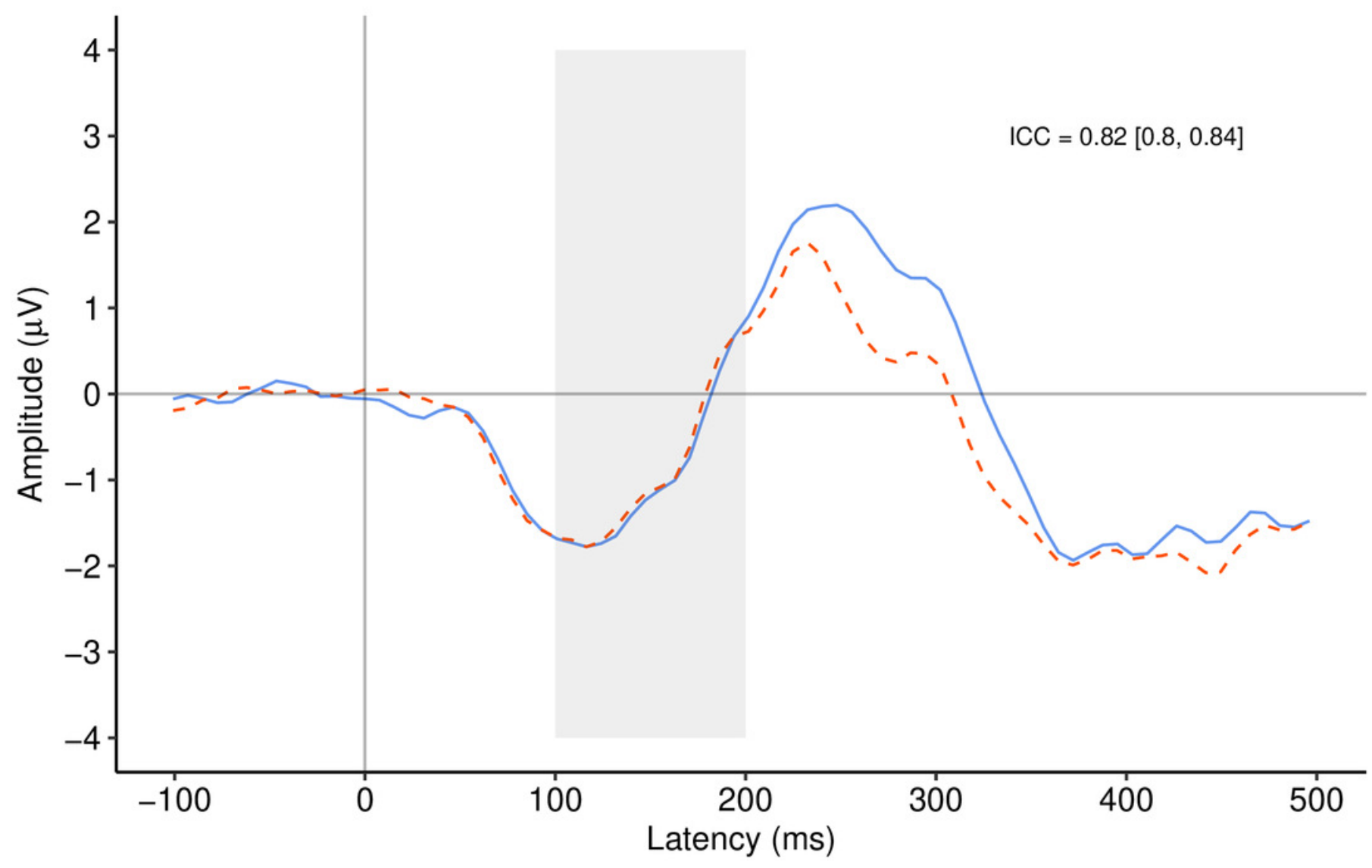




\section{Figure 5}

Topographic distribution of MMN and P300 ERP signals.

(A and B) Neuroscan and Flex MMN measured as the average signal between 66 and $166 \mathrm{~ms}$.

(C and D) Neuroscan and Flex P300 measured as the average signal between 198 and 298 ms. 
A

B

Neuroscan

Flex
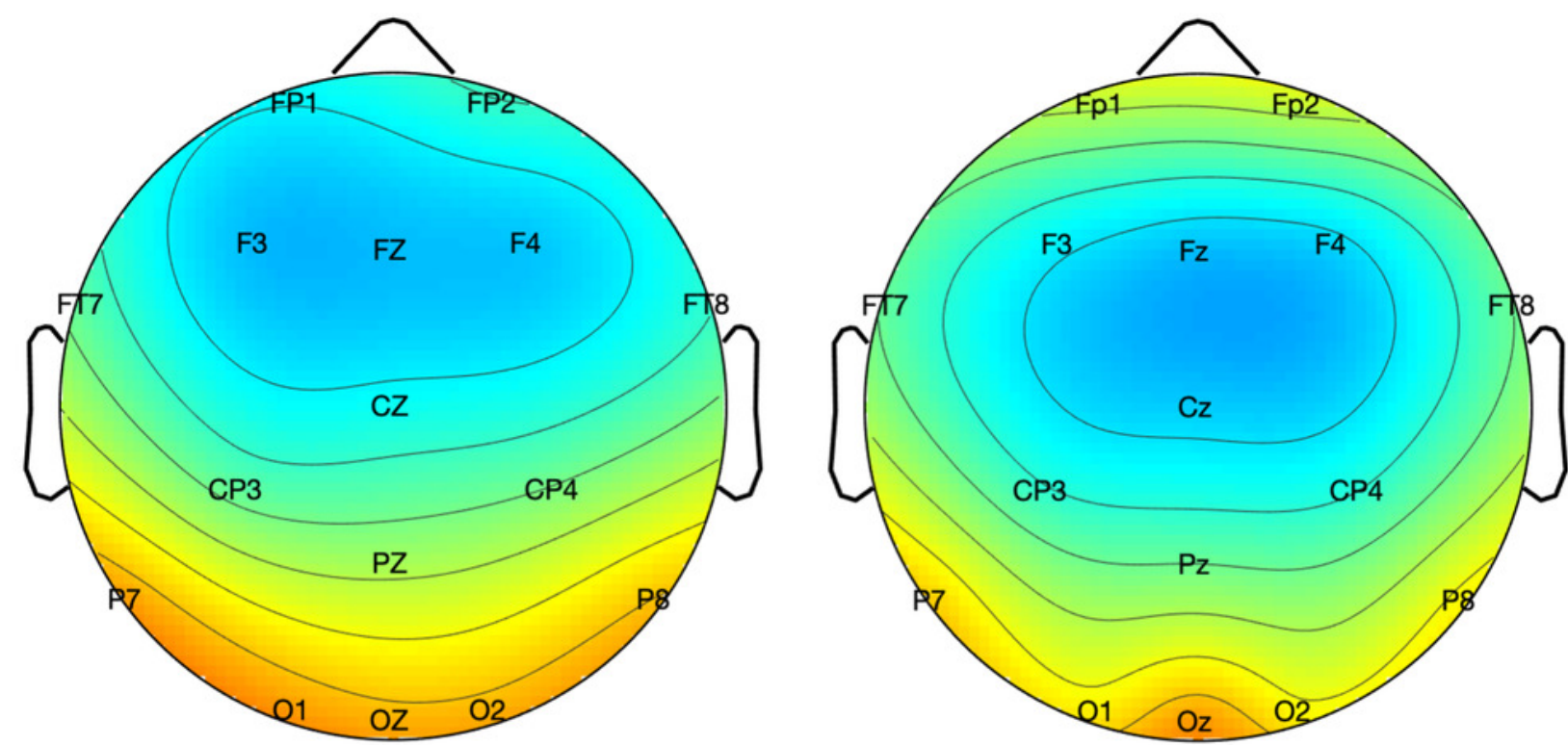

C
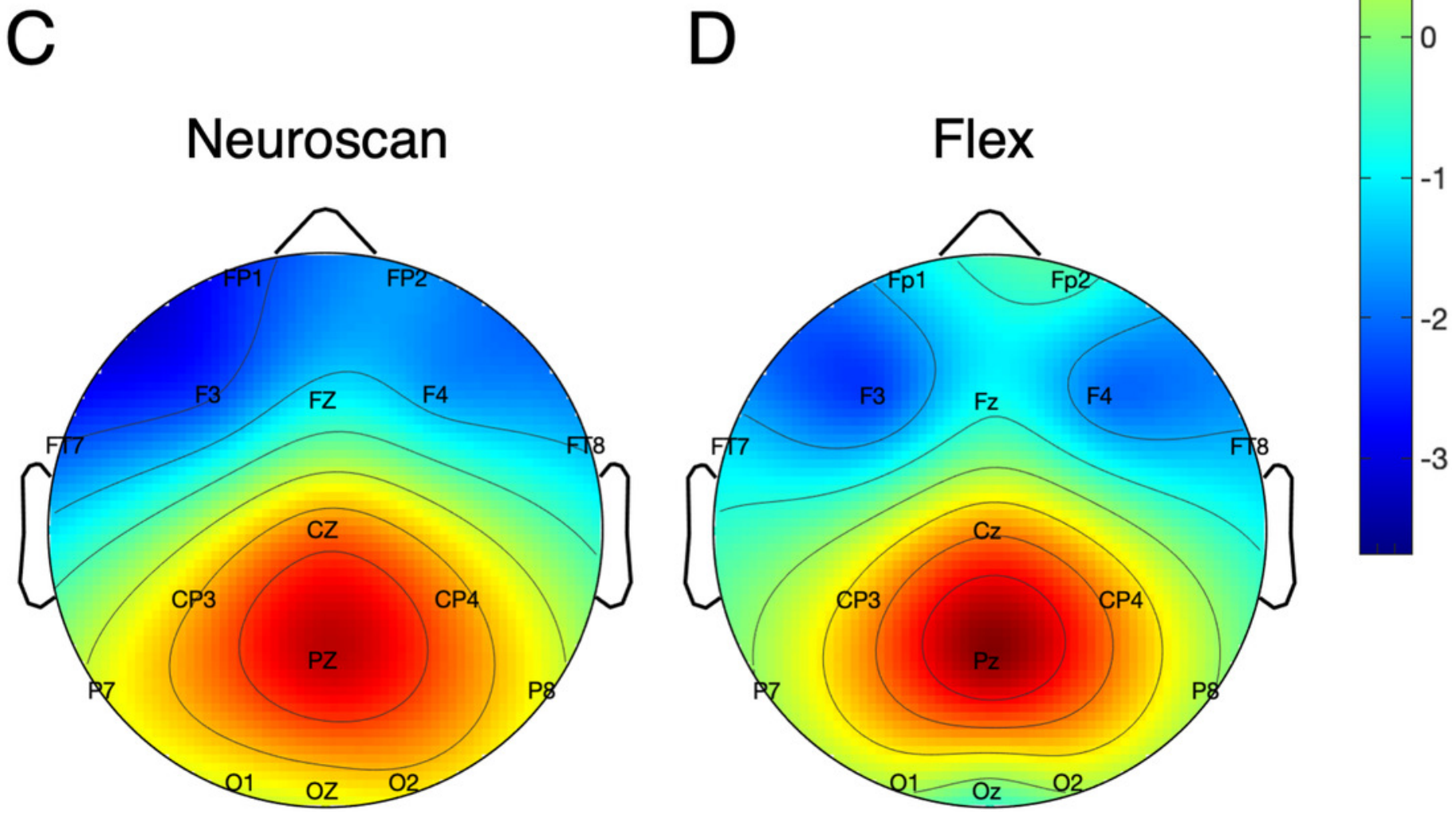


\section{Figure 6}

Group N170 ERP waveforms and intraclass correlations [95\% confidence interval] for Neuroscan and Flex systems by stimulus type.

( $A, C, E$, and $G$ ) depict left hemisphere waveforms (P7). (B, D, F, and H) depict right hemisphere waveforms (P8). Intraclass correlations were considered significant if the $95 \%$ confidence interval did not include zero. 


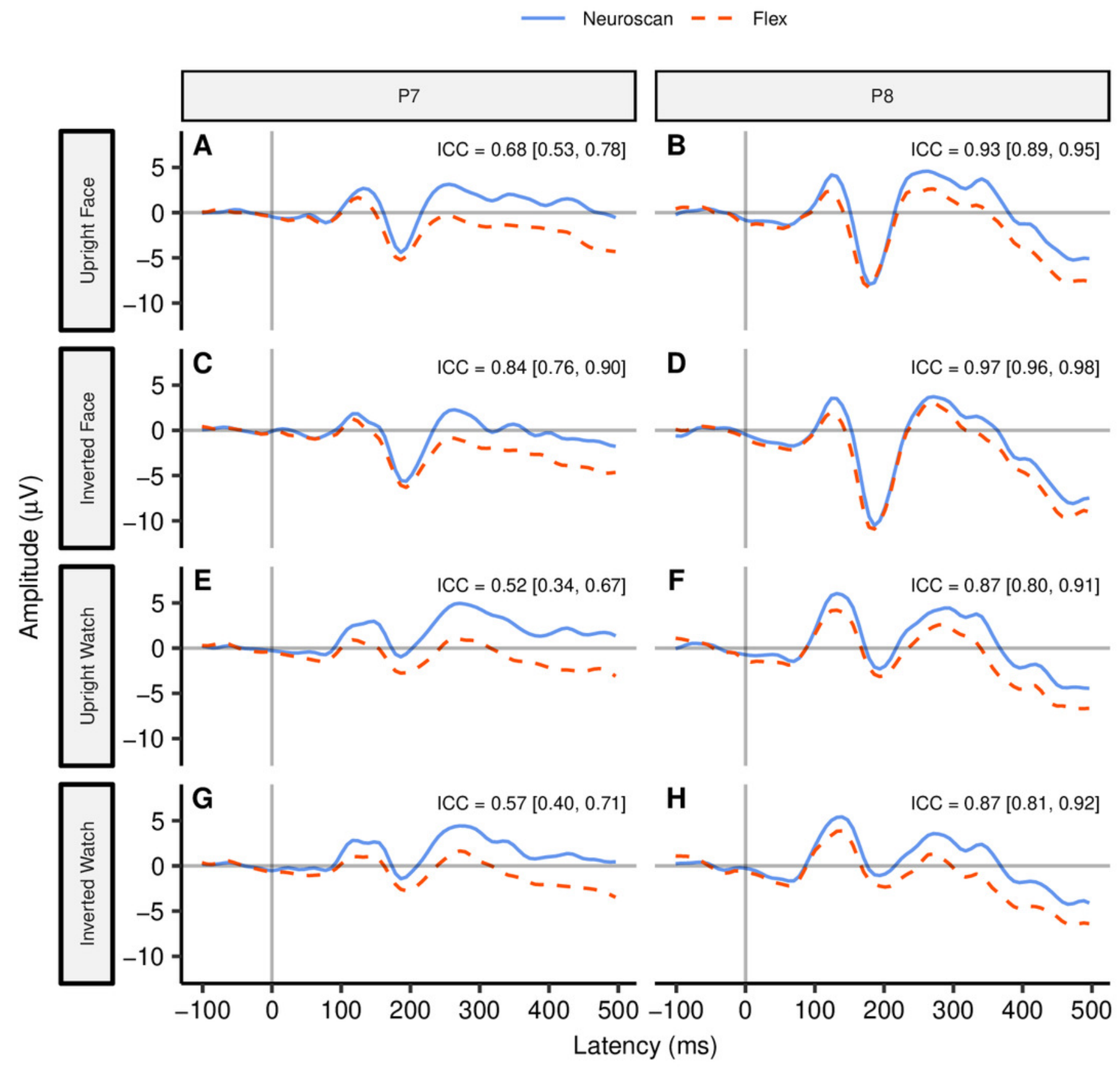


Figure 7

Topographic distribution of N170 ERP signals for each of the stimulus conditions.

Values represent the average signal between 162 and 200 ms. 
Neuroscan

Flex
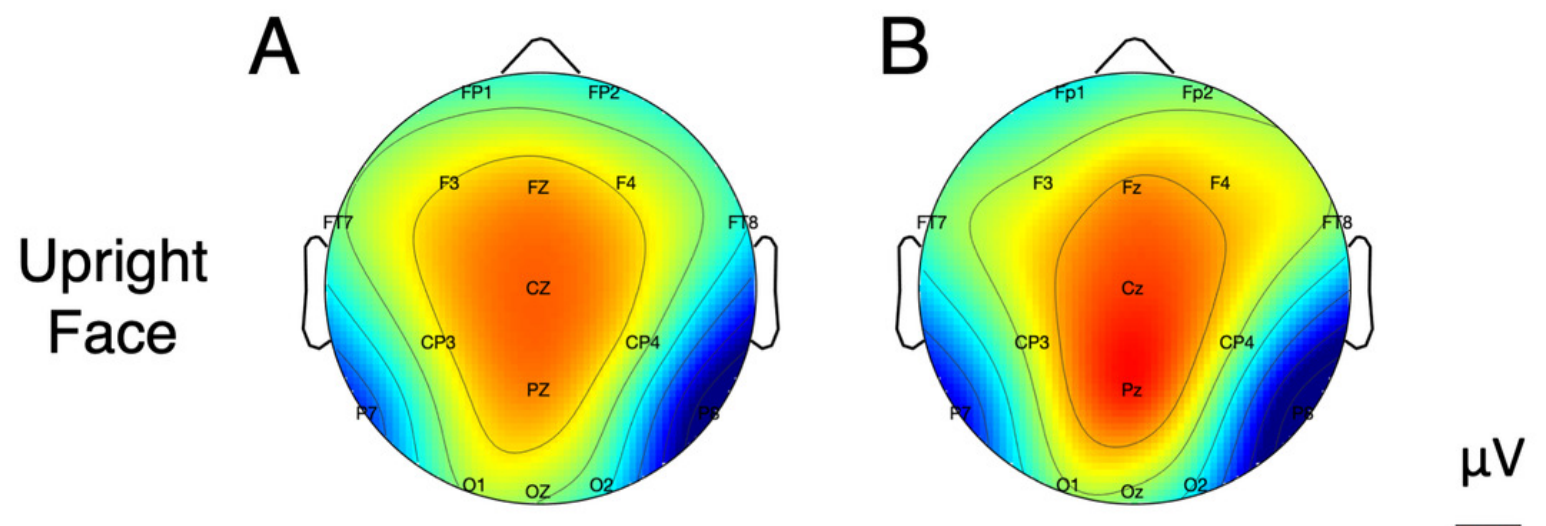

Inverted

C Face

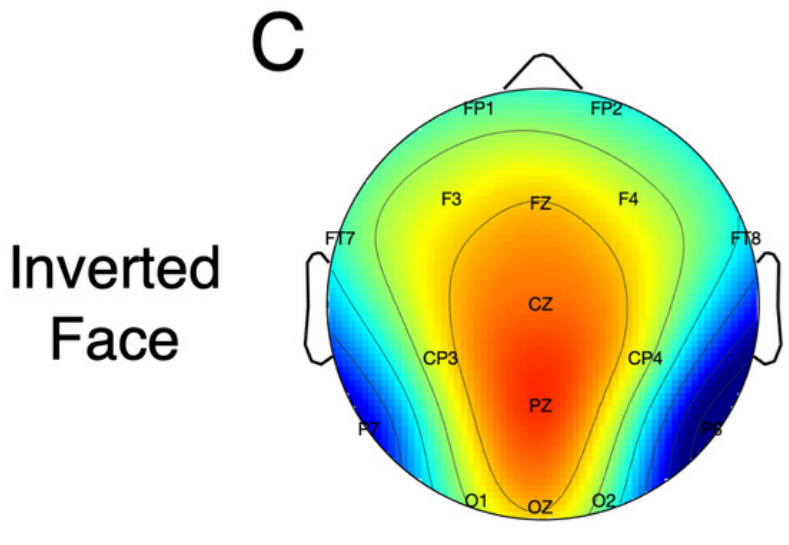

D

$\mathrm{E}$

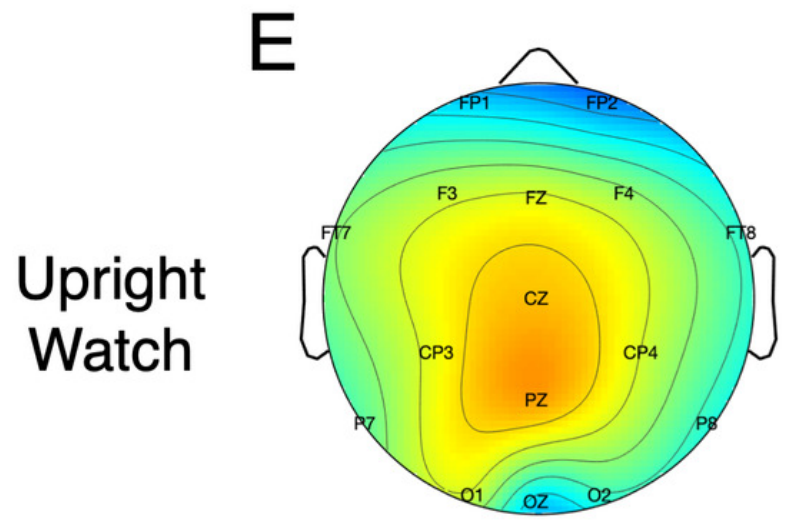

Upright Watch
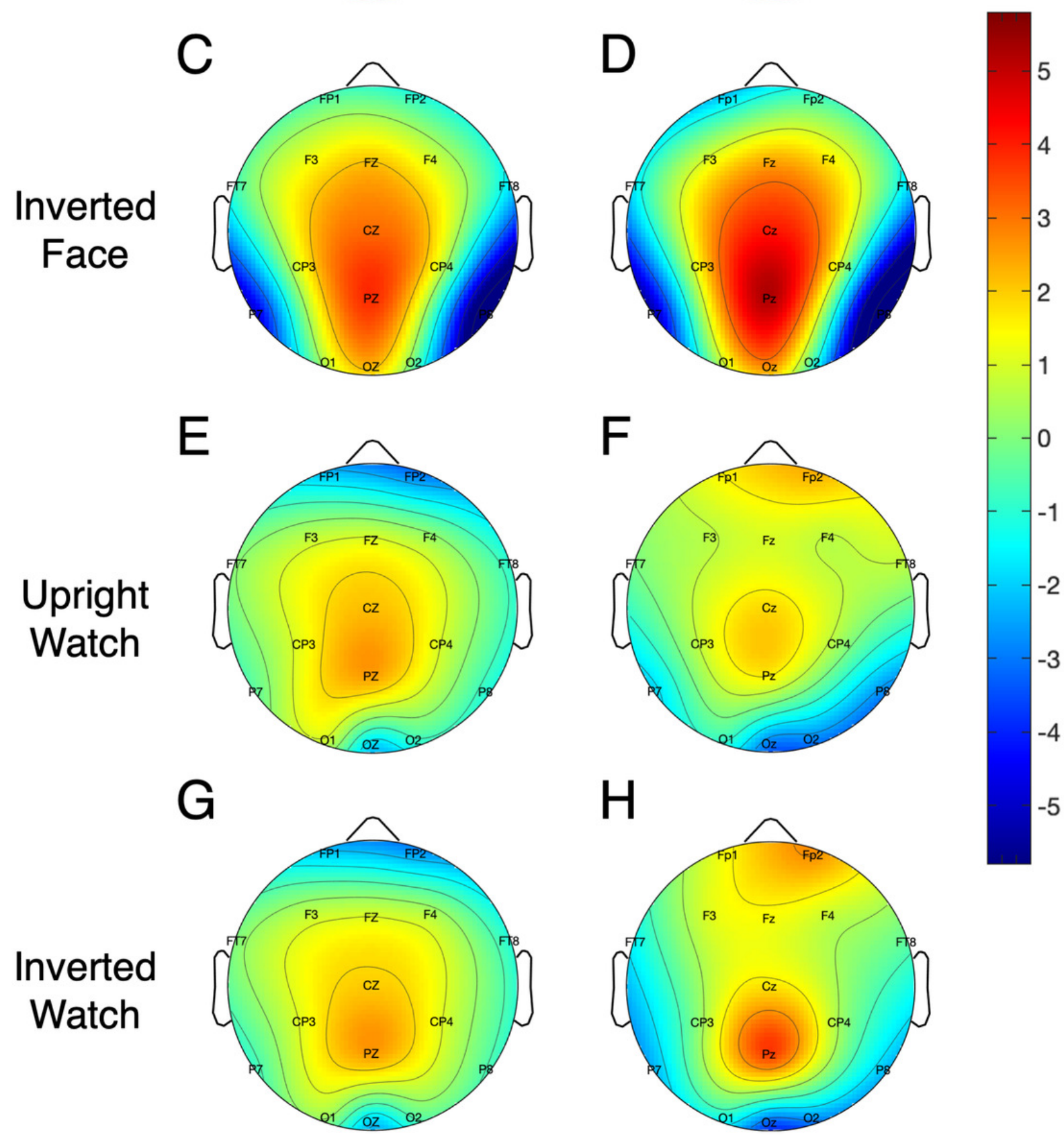
Figure 8

Signal-to-noise ratio (SNR) across frequency spectrum, $1-30 \mathrm{~Hz}$.

(A) Neuroscan SNR. (B) Flex SNR. SNR values were averaged across occipital electrodes (O1, $\mathrm{Oz}, \mathrm{O2}$ ) for each system. 


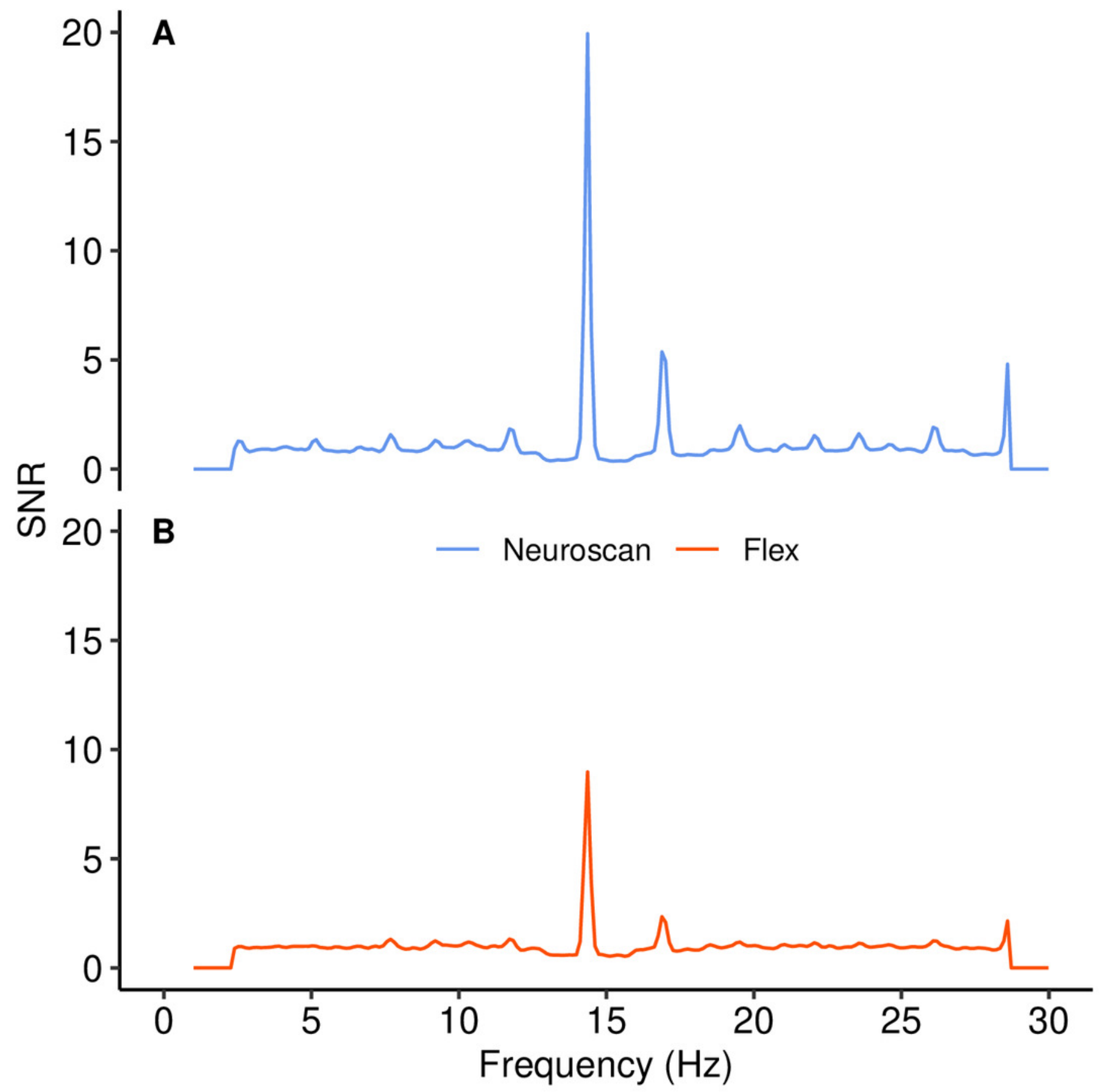


Figure 9

Resting state alpha band (8 - $12 \mathrm{~Hz}$ ) scalp topography maps.

(A) Neuroscan 'eyes open'. (B) Neuroscan 'eyes closed'. (C) Flex 'eyes open'. (D) Flex 'eyes closed'.
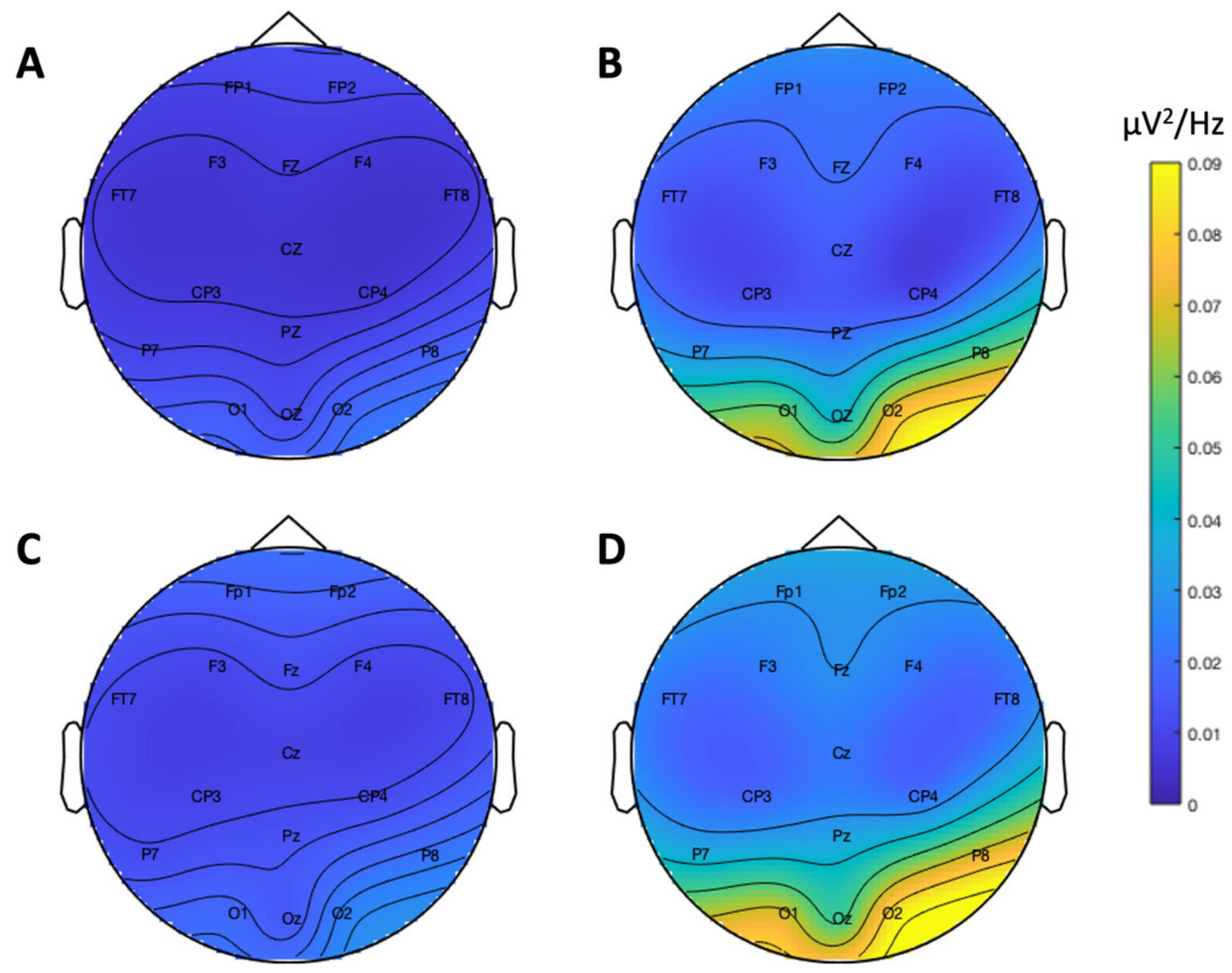
Figure 10

Power spectrum in 'eyes open' and 'eyes closed' conditions across frequencies (1 - 30 $\mathrm{Hz}$ ).

(A) Neuroscan power. (B) Flex power.

- Eyes Open - - Eyes Closed

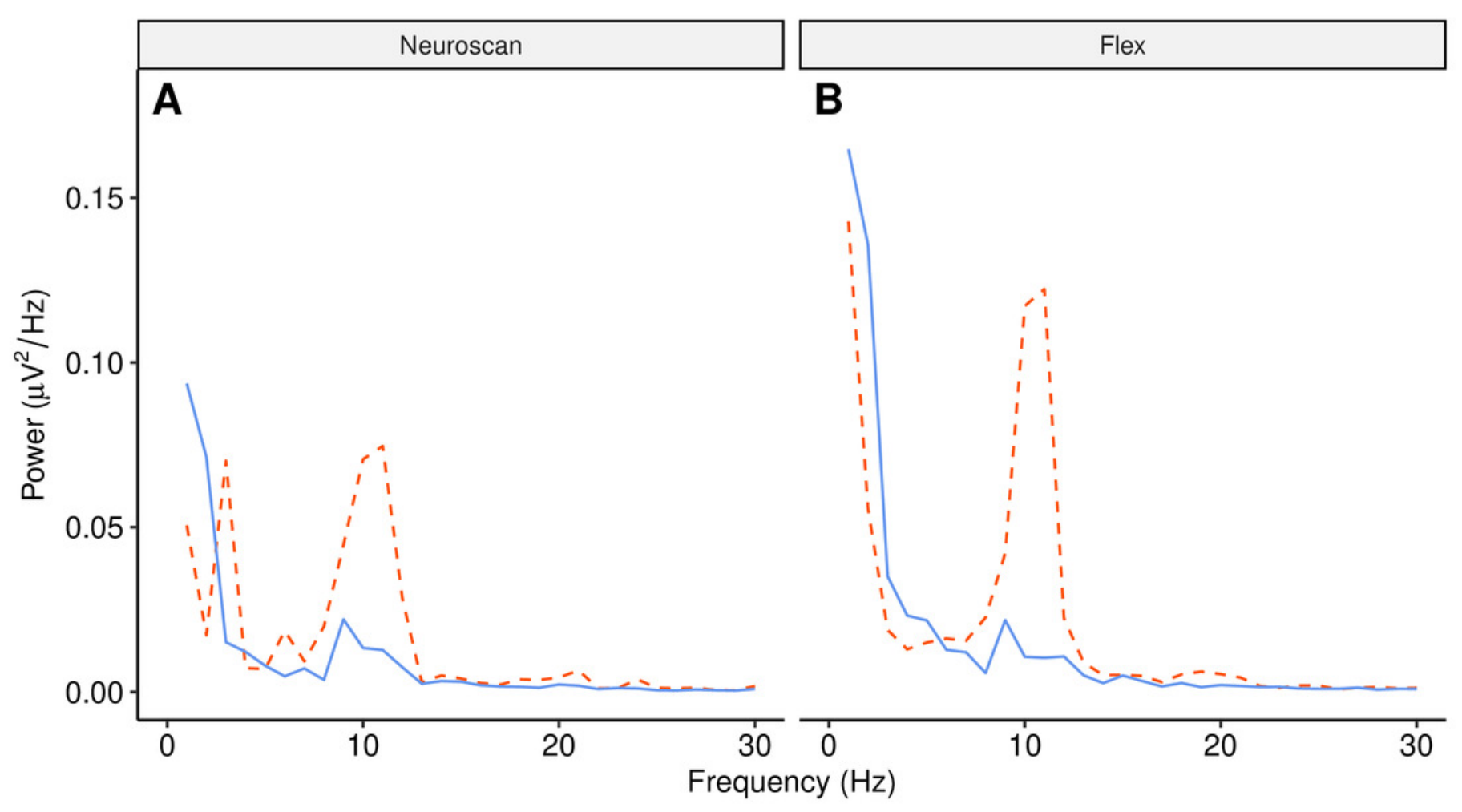




\section{Table $\mathbf{1}$ (on next page)}

Comparison of technical specifications of EEG systems. 
1

\begin{tabular}{|c|c|c|c|c|c|}
\hline & Neuroscan SynAmps & EPOC+ & EPOC Flex Saline & Muse 2 & Mindwave \\
\hline $\begin{array}{l}\text { Number } \\
\text { of } \\
\text { Channels }\end{array}$ & 64 & 14 & 32 & 4 & 1 \\
\hline $\begin{array}{l}\text { EEG } \\
\text { Electrode } \\
\text { locations }\end{array}$ & $\begin{array}{c}\text { User configurable. } \\
\text { International } 10 \text { - } 20 \\
\text { system }\end{array}$ & $\begin{array}{c}\text { Rigid. AF3, } \\
\text { F3, F7, FC5, } \\
\text { T7, P7, O1, } \\
\text { O2, P8, T8, } \\
\text { FC6, F8, F4, } \\
\text { and AF4 }\end{array}$ & $\begin{array}{c}\text { User } \\
\text { configurable. } \\
\text { International } 10 \\
\text { - } 20 \text { system }\end{array}$ & $\begin{array}{l}\text { Rigid. AF7, } \\
\text { TP9, TP10, } \\
\text { and AF8 }\end{array}$ & Rigid. FP1 \\
\hline $\begin{array}{l}\text { Reference } \\
\text { Locations }\end{array}$ & $\begin{array}{l}\text { User configurable } \\
\text { (online reference } \\
\text { and ground) }\end{array}$ & $\begin{array}{l}\text { TP9 and TP10 } \\
\text { (CMS and } \\
\text { DRL) }\end{array}$ & $\begin{array}{c}\text { User } \\
\text { configurable } \\
\text { (CMS and DRL) }\end{array}$ & $\begin{array}{l}\mathrm{FPz} \text { (CMS } \\
\text { and DRL) }\end{array}$ & $\begin{array}{l}\text { Earclip } \\
\text { (online } \\
\text { reference } \\
\text { and } \\
\text { ground) }\end{array}$ \\
\hline $\begin{array}{c}\text { Electrode } \\
\text { Material }\end{array}$ & $\mathrm{Ag} / \mathrm{AgCl}$ & $\begin{array}{l}\text { Saline-soaked } \\
\text { felt pad }\end{array}$ & $\begin{array}{l}\text { Saline-soaked } \\
\text { felt pad }\end{array}$ & $\begin{array}{c}\text { Silver } \\
\text { (frontal) } \\
\text { and } \\
\text { silcone } \\
\text { rubber } \\
\text { (temporal) }\end{array}$ & Silver \\
\hline Resolution & 24 bits & 14 or 16 bits & 14 bits & 10 bits & 12 bits \\
\hline $\begin{array}{l}\text { Sampling } \\
\text { rate }\end{array}$ & Up to $20 \mathrm{kHz}$ & 128 or $256 \mathrm{~Hz}$ & $128 \mathrm{~Hz}$ & $\begin{array}{l}220 \text { or } \\
500 \mathrm{~Hz}\end{array}$ & $512 \mathrm{~Hz}$ \\
\hline
\end{tabular}




\section{Table 2 (on next page)}

Number of excluded participants for each paradigm with reasons for exclusion.

If a participant was excluded from one EEG system (e.g., Neuroscan), then they were also excluded them from the other (e.g., Flex) 
1

\begin{tabular}{ccl} 
Paradigm & Number Excluded & \multicolumn{1}{c}{ Reason } \\
\hline MMN & 1 & Extra events in Neuroscan EEG file $(n=1)$ \\
P300 & 2 & $\begin{array}{l}\text { Greater than } 50 \% \text { rejected epochs }(n=1) \\
\text { Missing events in Emotiv EEG file }(n=1)\end{array}$ \\
N170 & 2 & $\begin{array}{l}\text { Greater than } 50 \% \text { rejected epochs }(n=1) \\
\text { Stimulus computer crash during task }(n=1)\end{array}$ \\
SSVEP & 1 & $\begin{array}{l}\text { Experimenter error with recording EEG }(n=1) \\
\text { Missing events in Emotiv EEG file }(n=1) \\
\text { Resting }\end{array}$ \\
& 2 & \begin{tabular}{l} 
Extra events in Neuroscan EEG file $(n=1)$ \\
\hline
\end{tabular}
\end{tabular}

2 


\section{Table 3(on next page)}

ERP signal quality of Neuroscan and Flex systems.

Median (inter-quartile range) [range] for number of rejected epochs and mean (standard error) [95\% confidence interval] for signal-to-noise ratios (SNR). Epochs were rejected if amplitude exceeded $\pm 150 \mu \mathrm{V}$. 
1

\begin{tabular}{|c|c|c|c|c|c|}
\hline \multirow[b]{2}{*}{ ERP } & \multirow[b]{2}{*}{ System } & \multicolumn{2}{|l|}{ Epochs removed } & \multicolumn{2}{|l|}{ SNR } \\
\hline & & Median (IQR) [range] & Z & Mean (SE) $[95 \% \mathrm{Cl}]$ & $\mathrm{BF}$ \\
\hline \multirow[t]{2}{*}{ MMN } & Neuroscan & $0(0)[0-47]$ & \multirow{2}{*}{$3.7^{*}$} & $4.15(0.41)[3.35,4.95]$ & \multirow{2}{*}{1.28} \\
\hline & Flex & $7.5(33.8)[0$ - 201] & & $3.54(0.26)[3.04,4.05]$ & \\
\hline \multirow[t]{2}{*}{ P300 } & Neuroscan & $0(0)[0-53]$ & \multirow{2}{*}{$2.8^{*}$} & $6.60(0.59)[5.43,7.76]$ & \multirow{2}{*}{50.79} \\
\hline & Flex & $4.5(20.5)[0-63]$ & & $5.10(0.38)[4.37,5.84]$ & \\
\hline \multirow[t]{2}{*}{ N170 } & Neuroscan & $0(0)[0-24]$ & \multirow{2}{*}{$1.6^{\perp}$} & $6.04(0.47)[5.11,6.97]$ & \multirow{2}{*}{7.84} \\
\hline & Flex & $0(6.0)[0-23]$ & & $5.12(0.29)[4.55,5.70]$ & \\
\hline
\end{tabular}

$2 \quad{ }^{*} \mathrm{p}<.01 ;{ }^{\perp} \mathrm{p}=.11$

3 


\section{Table 4 (on next page)}

Mismatch negativity (MMN) and P300 peak descriptive statistics for Neuroscan and Flex systems.

The MMN waveform was measured at Fz and calculated as the ERPs to deviant tones minus ERPs to standard tones in the passive condition. The pre-registered calculation represents the average signal from 100 - 200 ms whereas the post-hoc calculation represents the average signal from 66 - $166 \mathrm{~ms}$. P300 values were measured at Pz and represent the average signal from either 280 - 380 ms (pre-registered) or 198 - 298 ms (post-hoc) for deviant tones in the active condition. 
1

\begin{tabular}{llllll} 
Calculation method & Waveform & System & Mean & SE & $95 \%$ confidence interval \\
\hline Pre-registered & MMN & Neuroscan & -1.03 & 0.30 & {$[-1.61,-0.45]$} \\
& & Flex & -0.97 & 0.38 & {$[-1.71,-0.22]$} \\
\cline { 2 - 6 } & P300 & Neuroscan & 3.63 & 0.48 & {$[2.67,4.58]$} \\
& & Flex & 3.44 & 0.58 & {$[2.30,4.58]$} \\
\hline Post-hoc & MMN & Neuroscan & -1.40 & 0.25 & {$[-1.88,-0.92]$} \\
& & Flex & -1.40 & 0.35 & {$[-2.07,-0.72]$} \\
\cline { 2 - 6 } & P300 & Neuroscan & 3.22 & 0.43 & {$[2.38,4.07]$} \\
& & Flex & 3.56 & 0.51 & {$[2.57,4.55]$} \\
\hline
\end{tabular}

2 
Table 5 (on next page)

ERP signal-to-noise-ratios (SNRs). 
1

Peak

calculation

\begin{tabular}{|c|c|c|c|c|}
\hline method & ERP & Site & System & SNR (SE) [95\% CI] \\
\hline \multirow[t]{4}{*}{ Pre-registered } & MMN & $F Z$ & Neuroscan & $5.51(0.96)[3.64,7.39]$ \\
\hline & & & Flex & $4.76(0.71)[3.37,6.15]$ \\
\hline & P300 & $\mathrm{PZ}$ & Neuroscan & $11.3(2.01)[7.39,15.3]$ \\
\hline & & & Flex & $5.65(0.92)[3.85,7.44]$ \\
\hline \multirow[t]{4}{*}{ Post-hoc } & MMN & $\mathrm{FZ}$ & Neuroscan & $5.34(0.86)[3.67,7.02]$ \\
\hline & & & Flex & $4.82(0.78)[3.31,6.33]$ \\
\hline & P300 & $\mathrm{PZ}$ & Neuroscan & $10.2(1.73)[6.85,13.6]$ \\
\hline & & & Flex & $5.57(0.75)[4.11,7.04]$ \\
\hline \multirow[t]{16}{*}{ Pre-registered } & N170 - face upright & P7 & Neuroscan & $7.21(1.10)[5.05,9.36]$ \\
\hline & & & Flex & $6.90(0.88)[5.19,8.62]$ \\
\hline & & P8 & Neuroscan & $11.2(1.64)[8.01,14.5]$ \\
\hline & & & Flex & $8.44(0.97)[6.55,10.3]$ \\
\hline & N170 - face inverted & P7 & Neuroscan & $7.80(0.95)[5.94,9.66]$ \\
\hline & & & Flex & $6.33(0.72)[4.92,7.74]$ \\
\hline & & P8 & Neuroscan & $11.5(1.42)[8.68,14.3]$ \\
\hline & & & Flex & $10.5(1.25)[8.02,12.9\}$ \\
\hline & N170 - watch upright & P7 & Neuroscan & $7.67(1.80)[4.14,11.2]$ \\
\hline & & & Flex & $5.26(1.16)[3.00,7.53]$ \\
\hline & & P8 & Neuroscan & $8.63(1.54)[5.62,11.6]$ \\
\hline & & & Flex & $7.30(1.70)[3.97,10.60]$ \\
\hline & N170 - watch inverted & P7 & Neuroscan & $6.04(0.67)[4.74,7.35]$ \\
\hline & & & Flex & $6.61(1.31)[4.05,9.17]$ \\
\hline & & P8 & Neuroscan & $7.82(1.47)[4.95,10.7]$ \\
\hline & & & Flex & $5.07(0.97)[3.18,6.96]$ \\
\hline
\end{tabular}




\section{Table 6(on next page)}

N170 peak and latency group average descriptive statistics for left hemisphere (P7) and right hemisphere (P8). Figures represent mean values [95\% confidence interval].

N170 amplitude measures were calculated as the average amplitude between 120 and 220

ms. Latency measures were calculated as the time point corresponding to the minimum amplitude between 120 and 220 ms. 
1

\begin{tabular}{|c|c|c|c|c|c|}
\hline Site & Stimulus Type & Measure & System & Upright & Inverted \\
\hline \multirow[t]{8}{*}{ P7 } & Faces & Amplitude & Neuroscan & $-1.51[-2.40,-0.61]$ & $-2.84[-3.79,-1.89]$ \\
\hline & & & Flex & $-2.82[-3.93,-1.70]$ & $-3.69[-5.02 .-2.37]$ \\
\hline & & Latency & Neuroscan & $182[177,188]$ & $191[185,196]$ \\
\hline & & & Flex & $181[172.190]$ & $188[178,198]$ \\
\hline & Watches & Amplitude & Neuroscan & $0.58[-0.38,1.55]$ & $0.20[-0.83,1.24]$ \\
\hline & & & Flex & $-1.38[-2.55,-0.22]$ & $-1.57[-2.83,-0.31]$ \\
\hline & & Latency & Neuroscan & $178[166,189]$ & $178[167,190]$ \\
\hline & & & Flex & $176[162,189]$ & $182[170,194]$ \\
\hline \multirow[t]{8}{*}{ P8 } & Faces & Amplitude & Neuroscan & $-2.37[-3.78,-0.95]$ & $-3.43[-4.77,-2.09]$ \\
\hline & & & Flex & $-3.93[-5.65,-2.21]$ & $-4.56[-6.30,-2.81]$ \\
\hline & & Latency & Neuroscan & $181[176,186]$ & $188[183,193]$ \\
\hline & & & Flex & $178[173,184]$ & $184[179,190]$ \\
\hline & Watches & Amplitude & Neuroscan & $1.40[-0.17,2.97]$ & $1.43[-0.25,3.10]$ \\
\hline & & & Flex & $-0.54[-2.32,1.24]$ & $-0.16[-1.84,1.51]$ \\
\hline & & Latency & Neuroscan & $191[186,197]$ & $190[184,196]$ \\
\hline & & & Flex & $191[183,198]$ & $192[184,200]$ \\
\hline
\end{tabular}




\section{Table 7 (on next page)}

Signal-to-noise ratio (SNR) descriptive and inferential statistics.

SNR values represent average of occipital sites $(01,0 Z, 02)$. The values were considered significant if Z-scores were greater than $1.96(p<0.05)$. 


\begin{tabular}{|c|c|c|}
\hline System & Mean (SD) & Z-score \\
\hline Neuroscan & $19.94(9.51)$ & 12.47 \\
\hline Flex & $8.98(4.80)$ & 11.22 \\
\hline
\end{tabular}




\section{Table 8(on next page)}

Resting state alpha power descriptive statistics for each condition and system.

Power was measured as the power averaged over occipital sites $(01,0 z, 02)$ across frequencies $8-12 \mathrm{~Hz}$. 
1

\begin{tabular}{llccc} 
System & Measure & Mean & Standard error & 95\% confidence interval \\
\hline Neuroscan & Eyes Open & 0.01 & 0.00 & {$[0.01,0.02]$} \\
& Eyes Closed & 0.05 & 0.01 & {$[0.02,0.07]$} \\
Flex & Eyes Open & 0.01 & 0.00 & {$[0.01,0.02]$} \\
& Eyes Closed & 0.07 & 0.02 & {$[0.02,0.11]$} \\
\hline
\end{tabular}

2 